\title{
A DESCRIPTION OF THE LORENZ ATTRACTOR AT HIGH PRANDTL NUMBER
}

\author{
A.C. FOWLER \\ Room 2-336, Departinent of Mainematics, Massachusetts Institute of Technology, Cambridge, MA 02139, USA \\ and \\ M.J. McGUINNLSS \\ Department of Applied Mathematics, Room 317-50, California Institute of Technology, Pasadena, CA S1125, USA
}

Received 28 April $\| 981$

Rerised 1 April 1982

\begin{abstract}
We use the ideas of matched asymptotic expansions to construct a 'solution' or the Lorenz equations in the linit $r \sim \sigma \rightarrow \infty$. Unlike the case $r \rightarrow \infty, \sigma \sim 1$ which exhibits limit sycle behaviour, the solutions in the present case can be 'chaotic'; nevertheless, their structure can be analysed. Our sim is to obtain an aralytic approximation of the 'Lorenz map' which plots successive maxima of the variable $Z$. Particuli ly, we are able to expla in the peculiar cusp of this map. and indeed to predict the existence of many such cusps. It is our hupe that the methods and idieas presented here may be of use in elucidating the nature of other systems which exhibit chantic behaviour.
\end{abstract}

\section{Introduction}

The problem of the tri ssition to turbulence of shear flows at high r.eynolds number has perplexed many workers since the original paper of Reynolds [1], although our understanding of this phenomenon does slowly increase. Recent work by Orszag and Patera [2], for example, gives a clue as to how the fluid dynamics of Poiseuille flow may operate at transition. Subsequent discovery of other fiuid flows (Bénard convection, Taylor-Couette flow) which can appear, in some loosely defined sense, turbulent, has made the problem of fluid turbulence one of the most interesting and difficult of modern research.

Part of the difficulty lies in the virtual absence of (even approximate) constructive, analytical techniques for 'solving' the Navier-Stokes equations in such situations, and as a result, much interest has recently been shown in various kinds oi dynamical systems, which can also exhibit benaviour which is, in some sense, turbulent (chaotic, aperiodic).

Much of the driving force behind this whole approach to "turbulence" is the paper by Lorenz [3j, who, motivated by the possible indeterminacy of the state of the atmosphere, was led to the consideration of a set of three ordinary differential equations; these equations, derived by a three term Fourier series truncation of a model of thermal convection, have solutions which in certain circumstances are aperiodic. This observation of 'chaotic' (apparently random) global behaviour of the solutions suggests that the comparatively simple Lorenz model (three differential equations with only two

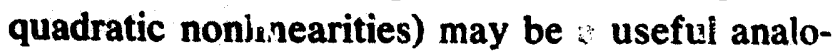
gue with which to study the more complicated equations governing fluid flow. $I_{h}$ particular, the Lorenz model exhibits 'int.mittency' (Manneville and Pomeau [4]), which was also observed by Reynolds in his experintents (a more recent discussion is that of Wygnanski and 
Champagne [5]). and is an important feature of the process of trarsition.

There has been a great deal of study of Lorenz's system. much of it numerical: see, for example. Morioka and Shimizu [6]. Analytically. McLa'ghlin and Martin [7] showed that the hifurcation of the non-trivial steady states which occurs as the "Rayleigh number" $r$ increases through a critical value $r_{c}$ is a subcritical Hopf bifurcation: this means that the bifurcating periodic solutions exist for $r<r_{c}$ and are unstable (which is why they are not observed). but offers no clue as to the actual evoluti.in of the solution.

The same dificulty occurs in plane Poiseuille How. At Reynolds numbers close to the critical value $\operatorname{Re}_{\mathrm{i}} \approx \mathbf{5 7 7 2}$. the amplitude of a spatially. independent modulation of the least stable travelling wave solution of the corresponding linearised problem satisfies

$\frac{d A}{d t}=k_{1} A+\left.k \cdot A\right|^{P} A$

where $A$ is the (suitably scaled) amplitude. The derivation of (1.1) by Stuart [8] uses the method of multiple scales: it can be extended to cover spatially-dependent disturbances (Stewartson and Stuart [9]). In (1.1), $k_{1}$ is proportional to (and of the same order as) $\operatorname{Re}-\operatorname{Re}_{\mathrm{c}}$. (1.1) is asymptotically valid for $|A| \sim\left|R e-R_{c}\right|^{1 / 2}$ in the formal limit $\operatorname{Re}-\operatorname{Re}_{i} \rightarrow 0, \operatorname{Re}_{i} \sim 1$ (i.e. fixed). This if $\operatorname{Re} k_{2}>0$ (as is the case for plane channel flow). sma!l amplitude solutions to (1.1) in which $|A| \sim\left|\operatorname{Re}-\operatorname{Re}_{\mathrm{c}}\right|^{1 / 2}$ exist if $\operatorname{Re} k_{1}<0$, i.e. $\operatorname{Re}<\mathbf{R e}_{i}$, bui these are unstable. If $\operatorname{Re}>\mathbf{R e}_{\mathrm{c}}$ $\left(\operatorname{Re} k_{1}>0\right)$, (1.1) predicts a 'burst', in which $|A|$ becomes large in finite time: at this point the asymptotic theory breaks down, and bifurcation theory is inadequate.

McLaughlin and Martin showed that, when (1.1) applies to bifurcations of the non-trivial steady states of the Lorenz equations, then also $\operatorname{Re} k_{i}>0$, ov that the bifurcation is sub-critical (periodic solutions exist and are unstabie for $\left.r<r_{c}\right)$, and pertarbation theory cannot trace the solutions beyond $|A| \sim\left|r-r_{\mathrm{c}}\right|^{1 / 2}$. Now si.ice $|A|$, $\left(\operatorname{Re} k_{1}\right)$ are small, and we can write (1.1) is

$\frac{1}{2} \frac{\mathrm{d}|A|^{2}}{\mathrm{~d} t}=k_{1 \mathrm{R}}|A|^{2}+k_{2 \mathrm{R}}|A|^{4}$

where $\mathbf{R}$ denotes the real part, it is possible (to the same order of approximation) to replace the derivative of the slowly varying amplitude by a discrete Euler a.pproximation. Specifically, since $A$ is the (complex) amplitude of an oscillatory perturbation of the exponential form $\exp [i \Omega t]$ we may take the time interval as $2 \pi / \Omega$. With $A_{n}=A(2 \pi n / \Omega),(1.2)$ is approximately

$\frac{\Omega}{4 \pi}\left[\left|A_{n-1}\right|^{2}-\left|A_{n}\right|^{2}\right]=k_{1 \mathrm{R}}\left|A_{n}\right|^{2}+k_{2 \mathrm{R}}\left|A_{n}\right|^{4}$,

i.e.

$\left|A_{n+1}\right|^{2}=\left[1+\frac{4 \pi k_{1 \mathrm{R}}}{\Omega}\right]\left|A_{n}\right|^{2}+\frac{4 \pi k_{2 \mathrm{R}}}{\Omega}\left|A_{n}\right|^{4}$,

which is of the form of a difference equation, valid locally for $\left|A_{n}\right|$ near zero.

More generally, one can consider bifurcation theory (multiple scales) as describing a local approximation to a difference equation which describes the relation between successive maxima (for example) of at least one of the variables. (It is clear that we can choose the time origin for $A_{n}$ to be such that the corresponding maxima in the perturbation occur at $t=2 \pi n / \Omega$ ). Denoting successive maxima by $M_{n}$, this means that locally near equilibria there is a one-one map $M_{n+1}=f\left(M_{n}\right)$ described asymptotically by the above procedure. In fact, if $M^{*}=f\left(M^{*}\right)$ is an equilibrium state of the equations, then for $\left|M_{n}-M^{*}\right| \ll 1$, we have

$$
\begin{aligned}
M_{n+1} \approx & f\left(M^{*}\right)+\left(M_{n}-M^{*}\right) f^{\prime}\left(M^{*}\right) \\
& +\frac{1}{2}\left(M_{n}-M^{*}\right)^{2} f^{\prime \prime}\left(M^{*}\right),
\end{aligned}
$$


whence

$$
\begin{aligned}
\left(M_{n+1}-M^{*}\right) \approx & f^{\prime}\left(M^{*}\right)\left(M_{n}-M^{*}\right) \\
& +\frac{1}{2} f^{\prime \prime}\left(M^{*}\right)\left(M_{n}-M^{*}\right)^{2}
\end{aligned}
$$

This is the equivalent of (1.3); bifurcation occurs as $f^{\prime}\left(M^{*}\right)$ (assumed positive) increases through 1 , and is supercritical if $f^{\prime \prime}\left(M^{*}\right)<0$ (convex, fig, 1a), and subcritical if $f^{\prime \prime}\left(M^{*}\right)>0$ (concave, fig. 1b).

Thus, locally, difference equations give one nreans of visual' ing bifurcations. However, one cannot expeci o be able to extend such difference equat. ins beyond the asymptotic region of validity $\left(\left|M_{n}-M^{*}\right| \ll 1\right)$, except perhaps in special circumstances. For example, consider the solution of a set of three ordinary differential equations for the variables $X, Y, Z$, in which we plot successive maxima of one of the variables, say $Z$. Equating $d Z / d t=0$, $\mathrm{d}^{2} Z / \mathrm{d} t^{2}<0$ gives a surface in three-space, each

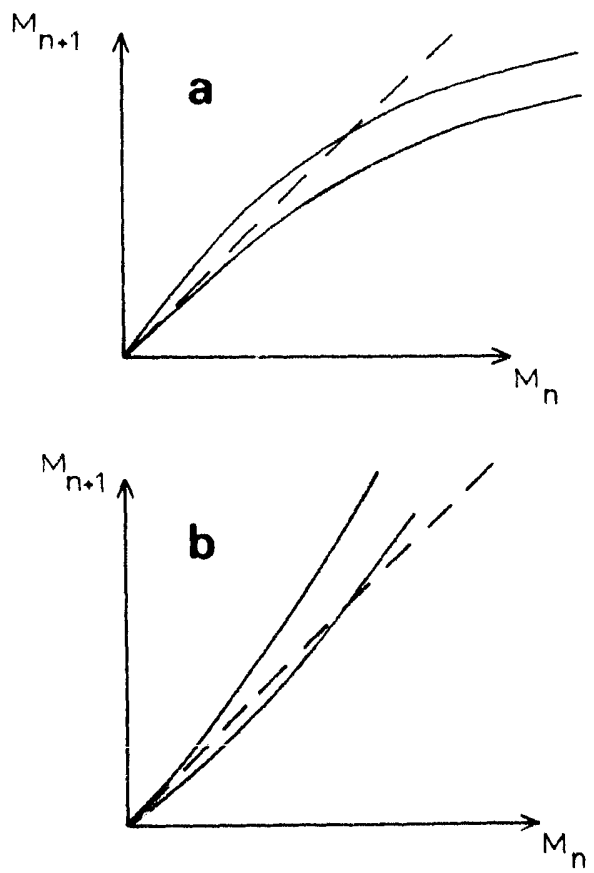

Fig. 1. Bifurcation of a non-trivial fixed point (corresponding to a periodic solution) from the origin as the slope there of the difference equation $M_{n} \rightarrow M_{n+1}$ increases through one. (a) Supercritical bifurcation, to stable fixed point. (b) Subcritical bifurcation; unstable limit cycle collapses and disappears as $\mathrm{dM}_{n+1} / \mathrm{d} M_{n}$ increases through one. point of which gives a possible maximum of $Z$, say $M_{n}$. If we take any particular value of $M_{n}$, then the intersc tion of the surface with $Z=M_{n}$ gives a line of points $(X(\alpha), Y(\alpha))$ for all of which $M_{n}$ is a maximum ( $\alpha$ is a parameter). Integration of the equations until the next intersection of the orbit with the surface of maxima gives the next maximum of $Z$, say $M_{n+1}$. However, it is clear that $M_{n+1}$ is a function of both $M_{n}$ and $\alpha$, and it is gererally most unlikely that the $\alpha$ dependence is negligible. If the system is dissipative (as that of Lorenz is) so that any attrasting invariant set has dimension less than 3 (at worst, two and a fraction, i.e. a surface crossed with a Cantor set), then on the attractor $M_{n+1}$ will in general be a function of $M_{n}$ and $\alpha$, where now $\alpha$ takes values not in a continuous interval, but in a Cantor set (which will depend on $M_{n}$ ). Thus the sequence of maxima $\left(M_{n}, M_{n+1}\right)$ plotted in Cariesian space will look like (part of) a one-dimensional map with multiple images - to each $M_{n}$ will correspond a Cantor set of values $\boldsymbol{M}_{n+1}$.

Despite this, Lorenz found that successive maxima of his variable $Z$ did indeed appear to lie on a curve, and that this curve (for the parametric values he used) had a prominent cusp. As a result, the modulus of the slope was everywhers greater than one, and consequently no fixed point or periodic orbit of the difference equation (and hence also the differential system) was stable. It is as a result of this behaviour that the system behaves chaotically. Successive maxima of $Z$ fluctuate erratically in time, and $X$ and $Y$ oscillate randomly on either side of the time axis. Chaos in difference equations has been extensively studied (for cxample $\mathrm{Li}$ and Yorke [10], May [11]), and is relatively well understood. Our point of view is therefore that the equations are essentially 'solved' once a prescription for such a diff rence equation can be given, and this is therefore the task we set ourselves. In some sense, a global difference equation of the type described above is an extension to large amplitudes of the local mul- 
tiple scale analysis, but we shall see that the method of approach is different.

Our methods will be constructive, hence approximate, but it is first worth enquiring how one ran approximate a 'function' which is not quite that. and why, even so. numerical solutions give a sequence of points which 'very nearly" lis on a curve. As we have mentioned above. the solutions of the Lorenz equation approach in $(X, Y, Z)$ space what we shall call the 'Lorenz attractor'. Ii is the product of a two-dimensional surface with a Cantor set. It is the contraction of 'clouds' of trajectories through different initial starting value to such an attracting surface which makes $M_{n+1}$ virtually independent of $\alpha$. To be more explicit, we can evaluate the 'divergence' of the flow. For the Lorenz model given in section 2. we find

$\frac{\partial \dot{X}}{\partial X}+\frac{\partial \dot{Y}}{\partial Y}+\frac{\partial \dot{Z}}{\partial Z}=-(\sigma+b+1)$

where $\sigma$ and $b$ are parameters, and a dot indicates differentiation with respect to time $T$. This indicates that volume: of initial points wecrease exponentially like $\exp [-(\sigma+b+1) T]$ : pirticulaily, we expect a lins of values $(X(\alpha)$. $\left.Y(a), M_{n}\right)$ for which $Z$ is maximal to have

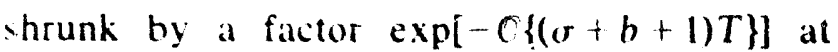
time $T$ later. If the $T$-interval between maxima is $C(1)$, then $\boldsymbol{M}_{n, 1}$ will not numerically depend on $\alpha$ provided $\exp [-\sigma\{(\sigma+b+1)\}]$ is small. Examination of Lorenz's solutions suggest that suciessive maxima are a time $T \approx 0.7$ apart. Further, he takes $\sigma=10, \quad b=8 / 3, \quad$ so $\exp [-c\{(r+b+1)\}] \approx 10^{4}$ is indeed small. This suggests why the difference equation of $M_{n+1}$ as a function of $M_{n}$ appears well defined.

However, we can go further. It seems reasonable that if the contraction rate were not small, then we should not see an apparently well-defined function, but rather more of the structure of the attractor (as for example in numerical stadies of the Hénon map (Hénon and Pomeau [12]). (This apparently occurs in the Lorenz model at higher values of the parameter $r$ (Lorenz, private communication).) It thus seems self-consistent that we only seek a difference equation for $M_{n}$ in the exac: context in which exp $[-(\sigma+b+1)]$ is small: this would seem to be when either $\sigma$ or $b$ is large. However, large $b$ is physically less meaningful, and we therefore choose to examine a limit in which $\sigma$ is large. As we shall show, it is then of mosi interest to choose $r$ large, and of order $\sigma$, since in this way, we are 'close' to the parameter regime in which the Hopf bifarcation, the strange invariant set, and strange attractor 'appear'. (We give the details in section 3.) With this assumption, observe that $\exp [-(\sigma+b+1)]$ is a transcendentally small term in $1 / \sigma$, and thus asymptotic expansions in powers of $1 / \sigma$ will not be able to differentiate between different 'leaves' of the attractor: it blurs them all together. This loss of information is in fact exactly what we want in order to determine a functional form of difference equation. Our statement will thus be that the asymptotic expansions we carry out will in principle determine the 'gross' structure of the attractor (that is, its general shape) without giving the small-scale topological structure. Clearly, from a physical point of view, the global structure is of primary interest.

We have another reason for considering th: asymptotic limit. In the common fluid mechanical situations wherein turbulent flows occur, transition often (perhaps normally) occurs at high values of the relevant dimensionless parameier (Reynolds number, Rayleigh number, Taylor number, etc.). Pipe flow has a lowest transition Rey'nolds number of $\approx 2300$, Bénard convection is initiated at a value (for free boundaries) of $\mathrm{Ra} \approx 657$ (and is turbulent at higher values), and so on. This suggests strongly that the solutions may have a boundary layer structure, which for finite amplitude motions should not be ignored. As suggested by Rosenblat and Davis [13], it is most probable that this may be a usefu! approach in treating transition 
in shear flows, and this is strongly supported by numerical experiments (Orszag and Patera [2]), and also laboratory experiments (well summarized by Schlicinting [14]) which show that the transition Reynolds number for pipe flow depends on the 'turbulence level' of the inlet disturbances. Thus it seems likely that the constructive approach to turbulent transition may well follow the same direction as that presented here, namely an explicit asymptotic construction of the solutions in the limit that the 'bifurcation' parameter tends to infinity. It is for all these reasons that we seek to analyse the Lorenz attractor at high values of the Prandi. number $\sigma$.

\section{The Lorenz model}

The Lorenz equations are

$$
\begin{aligned}
& \dot{X}=-\sigma(X-Y), \\
& \dot{Y}=(r-Z) X-Y, \\
& \dot{Z}=X Y-b Z,
\end{aligned}
$$

where a dot denotes differentiation with respect to time $T$. They were originally considered by Lorenz [3] as a crude (Fourier truncation) model of thermal convection in a rectangular box: their relevance to the original physical problem cannot be extended very far, roner it is the mathematical properties of (2.1) which are of major concern. In the original model, $\sigma, r$ and $b$ are positive constants which are respecil ely the Prandt: number of the fluid, a normalised Rayleigh number, and a measure of the aspect ratio of the box.

Lorenz analysed (2.1), and found that for $r<1,(0,0,0)$ is a unique stable steady state. For $r>1$, two non-trivial steady states $( \pm \sqrt{b(r-1)}, \pm \sqrt{b(r-1)}, r-1)$ bifurcate from the origin (which becomes unstable), and these new solutions are stable until

$$
r=r_{c}=\frac{\sigma(\sigma+b+3)}{\sigma-b-1}
$$

(if $\sigma>b+1$ ), when they too become (oscillatorily) unstable. McLaughlin and Martin [7] and Roshchin [16] showed that a subcritical Hopf bifurcation occurs at $r=r_{c}$, and so no stable small amplitude limit cycles are observed. At $\sigma=10, b=8 / 3, r=28$, Lorenz found numerical evidence of aperiodic motion. In phase space, the orbits tend towards a suriace called a strange attractor, on which sucil motions can occur. Later investigations (e.g. Yorke and Yorke [20]) showed that attracting aperiodic motion begins (wh'n $\sigma=10, b=8 / 3$ ) at $r \approx$ 24.06, whereas the Hnpf bifurcation does not occur until $r \approx 24.74$.

Subsequent numerical studies (Shimizu and Morioka [15], Manneville and Pomeau [17], Morioka and Shimizu [6]) indicate that for fixed $\sigma$ and $b$, this aperiodic behaviour alternatively undergoes successive transitions to periodic behaviour, more aperiodic behaviour, and so on, as $r$ increases. Eventually, for sufficiently high $r$, the system remains in a periodic state. This limit cycle which emerges at high $r$ is due to the system behaving as a weakly nonlinear oscillator, and its form was successfully andlysed by Robbins [18] and Shimizu [19] using perturbation methuds.

Perhaps the most important and far reaching insight of Lorenz into the behaviour of the aperiodic solutions was his plotting of successive maxima of one of the variables, $Z$ : thus if $M_{n}$ is the $n$th maximuin of $Z$ in any computer output of the solution, Lorenz plotted successive pairs $\left(M_{n}, M_{n+1}\right)$ and found that, instead of a random scatter, $M_{n+1}$ appeared to behave as a function of $M_{n}$. The reasons for this have already been mentioned in section 1 . Importantly, if the difference equation derived numerically is used as a predictor of the future state of the sys.em, Lorenz showed that the solution would behave erratically and aperiodically, since the sucussive maxima of $Z$ would wander 'randomly' along the $\left(M_{n}, M_{n+1}\right)$ curve. The $\left(M_{n}, M_{n+1}\right)$ curve (or Lorenz map) ot ained by Lorenz has the form shown sche- 
matically in fig. 2. It is possible to identify the cusp as the source of the aperiodicity, since it is this which causes the slope to be non-monotone. A natural question is thus to ask, why does this difference equation have such a cusp? Already, Lorenz [3] knew that maxima near the cusp were obtained after extrismely close approaches to the origin. Yorke and Yorke [20] used a knowledge of the linearised behaviour near the origin to give an approximate form of the difference equation near the cusp (which agrees with our results below): their analysis also agreed reasonably well with numerical results.

Our aim in this paper is to give an answer to this question by constructing an approximate Lorenz map explicitly, and show that it has the desired cusp-like feature. In so doing, we elucidate the nature of the solutions, and show what facets of their behaviour are responsible for the cusp. and hence the 'turbulent' behaviour. We hope that in this way we may shed light on the Lorenz model, and also by extension on other systems in winch chaotic fluctuations occur.

The rest of the paper is laid out in such a manner as to reflect the chronological progress of the work, which may facilitate an understanding of the material. In section 3 we re-scale the equations, and discuss the qualitative form

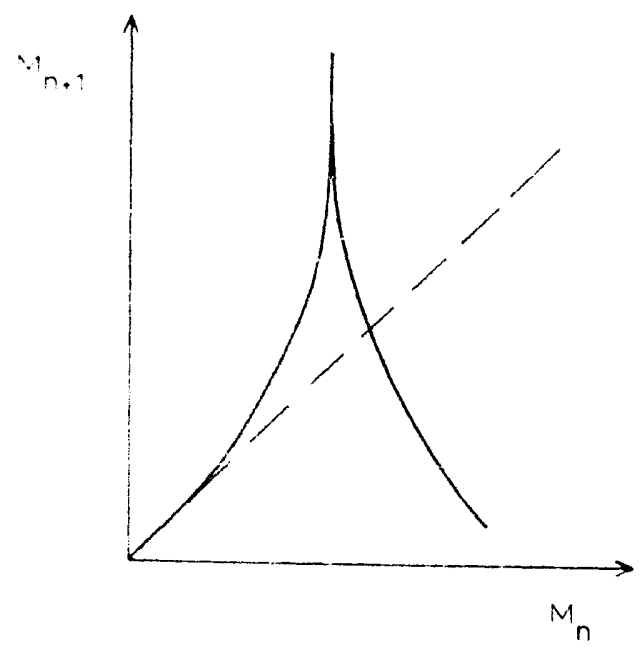

Fig. : Schematic form of difference equation $M_{n+1}=f\left(M_{n}\right)$ obtained by Lorenz. The dashed line here (as elsewhere) is $M_{n+1}=M_{n}$. of the solutions on this basis. The detailed asymptotic analysis leading to a difference equation is carried out in section 4 . The structure of this difference equation is studied in section 5. A comparison of the predictions from this theoretical difference equation with actual numerical results follows in section 6 . A discussion of the work follows in section 7 .

\section{Scaling of the equations and nature of the solutions}

The two topics of this section may be treated in any order. Though less logical, it is perhaps clearer if we scale the equations first, before describing the nature of the solutions. To do so, we recall that the Lorenz equations are

$$
\begin{aligned}
& \dot{X}=-\sigma X+\sigma Y, \\
& \dot{Y}=(r-Z) X-Y, \\
& \dot{Z}=X Y-b Z,
\end{aligned}
$$

where a dot signifies differentiation with respect to the dimensionless time, $T$. The non-trivial steady states

$$
X=Y= \pm \sqrt{b(r-1)}, \quad Z=r-1 \text {, }
$$

become unstable at $r=r_{\mathrm{c}}$, where

$r_{\mathrm{c}}=\frac{\sigma(\sigma+b+3)}{\sigma-b-1}$

In seeking to examine the nature of the bifurcating behaviour at large $\sigma$, we observe that $r_{\mathrm{c}} \sim \sigma$, and hence it is natural to let $r \sim \sigma$; thus we define

$r=\rho \sigma, \quad \rho \sim \sigma(1)$ as $\sigma \rightarrow \infty$.

It is clear when $r$ and $v$ are both large, that $X$ and $Y$ undergo rapid change in times $T$ of $\mid O(1 / \sigma)$. To isolate this time scale, we define the new time 
variable $t$ by

$t=\sigma \mathbf{r}$

Finally, to choose $X, Y$ and $Z$ scales we observe that it is natural to choose $Z \sim r$ in (3.1) $)_{2}$ then the $X$ and $Y$ scales are chosen (the same) so that (3.1), has an $O(1)$ forcing term when $t \sim 1$ : this leads to

$X=\sqrt{r \sigma} x, \quad Y=\sqrt{r \sigma} y, \quad Z=r z$.

Substituting (3.4), (3.5) and (3.6) into (3.1) leads to the scaled equations

$$
\begin{aligned}
& \frac{d x}{d t}=-x+y, \\
& \frac{d y}{d t}=\rho x(1-z)-\gamma y, \\
& \frac{d z}{d t}=x y-\delta z,
\end{aligned}
$$

where the parameters $\gamma$ and $\delta$ are defined by

$$
\gamma=\frac{1}{\sigma}, \quad \delta=\frac{b}{\sigma}
$$

In considering $r \sim \sigma \gg 1, b \sim 1$ in (3.1), we equivalently have

$\rho \sim 1, \quad \gamma \sim \delta \rightarrow 0$.

The analysis to follow recuires only that $\delta \ll 1$, and we may allow $\gamma \sim 1$, although for convenience we shall usually assume $\gamma \sim \delta$. The relevance of this is that $\delta$ can be made small either by letting $\sigma \rightarrow \infty$, or equivalently $b y$ holding $\sigma \sim 1$, and letting $b \rightarrow 0$ (also with,$\sim 1$ ): in Lorenz' original scheme, this corresponds to taking a convection cell of small ar pect ratio. We later show that the analysis in both these cases $(b \rightarrow 0$ or $b \rightarrow \infty)$ is consistent with the idea that the volume contraction of c ouds of trajectories between successive $m 8$ sima of $Z$ is large; that is (recalling section 1), if $T^{*}$ is a typical time between maxima of $Z$, then the contraction ratio $\exp \left[-(\sigma+b+1) T^{*}\right]$ is transcendentally small if $\delta \ll 1$ and $\gamma \leqslant 1$. For Lurenz' original values, we have $b=8 / 3, \sigma=$ 10, whence $\delta=1 / 4$. Though hardly infinitesimal, nevertheless the $\left(\boldsymbol{M}_{n}, \boldsymbol{M}_{n+1}\right)$ plots form a curve, and the time behaviour of $x, y$, and $z$ resembles that described below. This signifies that the smallness of $\delta$ gets exponentially 'amplified' by the equations, so that the form of the approximate solutions is valid for values of $\delta$ which are not numerically infinitesimal.

Let us now consider the system (3.7). Suppose that $x$ and $y$ are quite small at some point (e.g. $O(\delta)$ ); then (3.7) $)_{3}$ implies $z \approx$ constant on times $t \sim 1$. In this case $(3.7)_{1}$ and $(3.7)_{2}$ constitute a linear system for $x$ and $y$ :

$$
\left(\begin{array}{l}
\dot{x} \\
y
\end{array}\right)=\left(\begin{array}{cc}
-1 & 1 \\
\rho(1-z) & -\gamma
\end{array}\right)\left(\begin{array}{l}
x \\
y
\end{array}\right) \text {. }
$$

(3.10 has solutions probortional to $\exp (\lambda t)$, where

$$
\lambda=\frac{1}{2}\left[-(1+\gamma) \pm\left\{(1+\gamma)^{2}+4[\rho(1-z)-\gamma]\right\}^{1 / 2}\right]
$$

roughly speaking, then, $x$ and $y$ sither grow exponentially or decay exponentially (or oscillat ,rily! depending on whether $z<: 1-\gamma / \rho$ or $w_{\text {, ther }} z>1-\gamma / \rho$. (Since $\gamma / \rho=1 / r$. we may a sume $1-\gamma / \rho>0$ since $r>1$ for the non-trivial s. sady states to exist.) Now if $z>1-\gamma / \rho$, then $x$ and $y$ (which were small anyway) decay exponentially fast in $t$. Thus $x y$ becomes very small, and so $(3.7)_{3}$ implies that $\dot{z} \approx-\delta z$. Thus $x$, $y$ continue to approach zero while $z$ decays exponentially like exp(-st). Eventually, $z<$ $1-\gamma / \rho$, and $x$ and $y$ begin to grow: but they are $\exp [-\sigma(1) / \delta]$, hence they remain small during a further time $t \sim O(1 / \delta)$, during which $z$ decreases further. Finally, $x$ and $y$ (growing exponentially) become very quickly (in $t \sim 1$ ) $O(1)$, and suddenly $x, y, z$ all change in $t \sim C(1)$. 

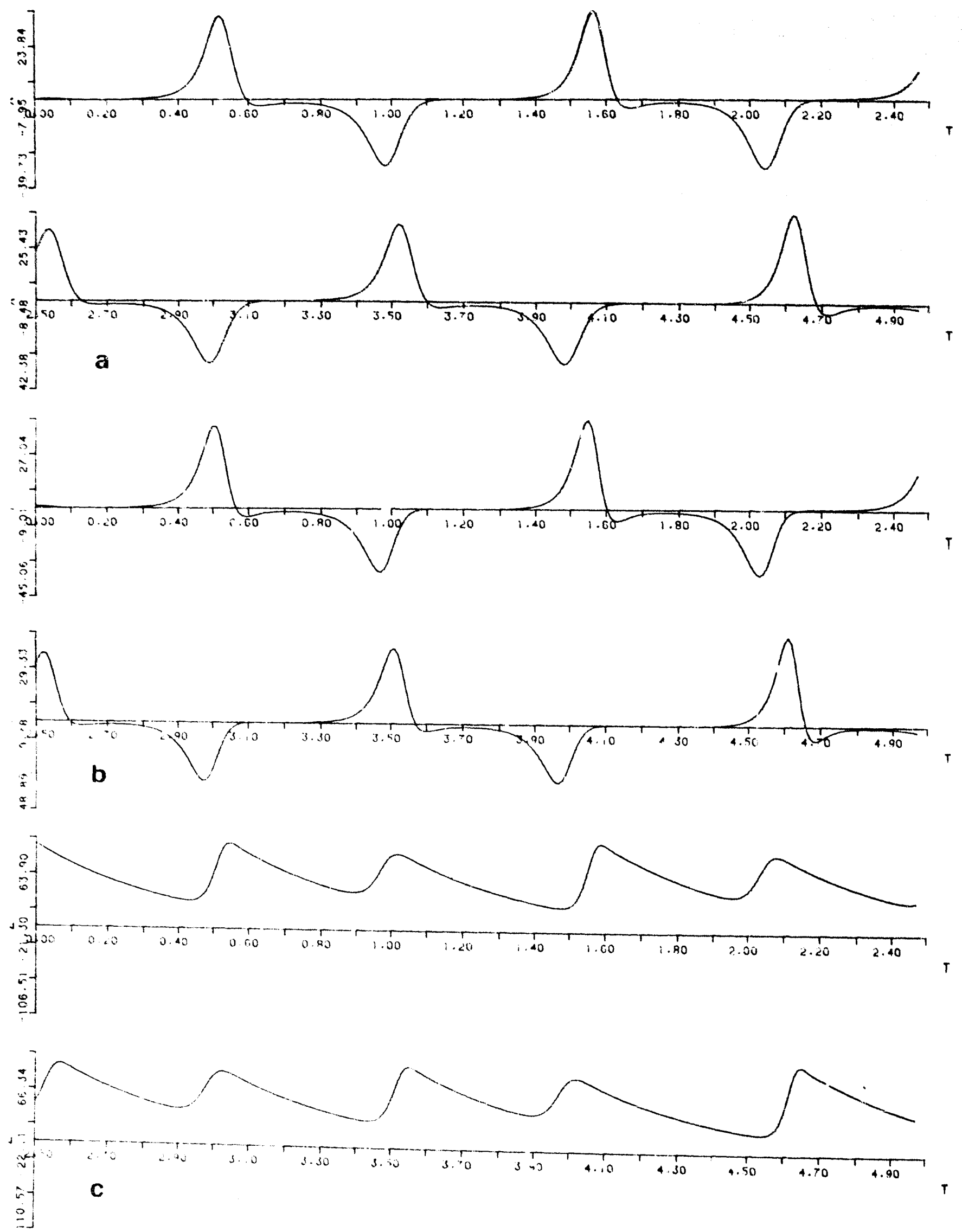

Fig. 3. Nunerical solution of the Lorenz equations (3.1) at parameter values $\sigma=50, r=70, b=1$. 
Supposing $x y>0$ (as follows from (3.7), , then $z$ is rapidly increased to a value $>1-\gamma / \rho$, and thus $x$ and $y$ rapidly decrease again, and the cycle is repeated. This sequence of events is portrayed in fig. 3, which is actually the result of a numerical integration of the equations. The increase in $z$ occurs slightly later than the $x$ and $y$ increase, since it is the latter to which $z$ responds.

The above description gives the clue as to how to analyse the solution: the motion consists of two distinct parts, a fast pulse-like phase and a slow relaxing phase, during which the controlling variable $z$ slowly ages. This suggests that singular perturbation theory may be a useful tool, as in the analogous behaviour of relaxation oscillations (e.g. Cole [21]). The only point here is that we do not constrain the solutions to be periodic (since generally they need not be), whereas in Cole's treatment of the relaxing Van der Pol oscillator this was assumed (from the theory). Cole's treatment could equally well be applied to give a difference equation relating successive maxima, but this equation would necessarily have a single stable fixed point. Such an extension would be analogous to using multiple scales in bifurcation problems, rather than the Poincaré-Lindstedt method, which gives only the periodic solution, and not the approach to it.

We note, if the arove description is correct, that the time between pulses may be written $t=\tau^{*} / \delta, \tau^{*} \sim \mathcal{O}(1)$, and hence the contraction ratio between pulses is $\exp [-(\sigma+b+$ 1) $\left.\tau^{*} / \sigma \delta\right]=\exp \left[-(\sigma+b+1) \tau^{*} / b\right]$. With $b \leqslant 1$, $\sigma \geqslant 1$, this ratio is small precisely when $\delta \ll 1$, and is in fact $\exp [-O(1) / \delta]$, as stated. We find $\tau^{*} \sim 1$, as assumed, in section 4 .

\section{A difference equation for the Lorenz system}

\subsection{Fast pulse}

Henceforth, we suppose $\gamma \sim \delta$, let us say

$$
\gamma=\bar{\gamma} \delta, \quad \bar{\gamma}=1 / b=O(1) \text {. }
$$

This assumption corresponds to considering $r \sim \sigma \rightarrow \infty, b \sim \mathcal{O}(1)$. The equations to be studied are thus

$$
\begin{aligned}
& \dot{x}=-x+y, \\
& \dot{y}=\rho x(1-z)-\delta \bar{y} y, \\
& \dot{z}=x y-\delta z .
\end{aligned}
$$

We first seek approximate solutions in the fast pulse, where $x, y, t \sim 1$ (the time origin will be specified in a moment). We sulistitute expressions $x \sim x_{0}+\delta x_{1}+\cdots$, etc., in (4.2) to obtain

$$
\begin{aligned}
& \dot{x}_{0}=-x_{0}+y_{0}, \\
& \dot{y}_{0}=\rho\left(1-z_{0}\right) x_{0}, \\
& \dot{z}_{0}=x_{0} y_{0} ; \\
& \dot{x}_{1}=-x_{1}+y_{1}, \\
& \dot{y}_{1}=\rho\left(1-z_{0}\right) x_{1}-\rho z_{1} x_{0}-\bar{\gamma} y_{0}, \\
& \dot{z}_{1}=x_{1} y_{0}+x_{0} y_{1}-z_{0} ;
\end{aligned}
$$


and so on. The 'initial conditions' for $x, y, z$ are that they should match into the preceding slow phase,

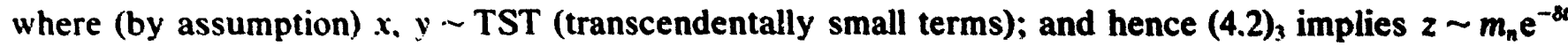
with transcendentally small error. We will suppose $m_{n}$ is independent of $\delta$ (this is not a serious restriction). Then the appropriate matching conditions for $x, y, z$ are

$$
\begin{aligned}
& x_{0} \rightarrow 0, \quad y_{0} \rightarrow 0, \quad z_{0} \rightarrow m_{n} \quad \text { as } t \rightarrow-\infty, \\
& x_{1} \rightarrow 0, \quad y_{1} \rightarrow 0, \quad z_{1}+z_{0} t \rightarrow 0 \quad \text { as } t \rightarrow-\infty,
\end{aligned}
$$

and so on. The aim of the analysis is to relate any given $m_{n}$ to the appropriate value $\left(m_{n+1}\right)$ for the beginning of the next fast pulse.

Solutions of (4.3) are

$$
\begin{aligned}
& x_{0}=\dot{\phi} / \sqrt{\rho}, \\
& y_{0}=-(k / \sqrt{\rho}) \sin \phi, \\
& z_{0}=1+(k / \rho) \cos \phi,
\end{aligned}
$$

where

$$
k=\rho\left(1-m_{\eta}\right),
$$

and $d$ satisfies

$$
\ddot{\phi}+\dot{\phi}+k \sin \phi=0 .
$$

The matching conditions require $\phi \rightarrow \pi$ as $t \rightarrow-\infty$, and we are at liberty to specify the time origin to be such that

$$
\pi-\phi \sim \pm \exp \left[\frac{1}{2}\left\{-1+(1+4 k)^{1 / 2}\right\} t\right], \quad t \rightarrow-\infty
$$

plus and minus signs corresfond to $x$ being negative and positive within the pulse: we select the plus sign withou: loss of generality (since the equations are unchanged if $x$ and $y$ change sign). Note that the easy first integral $y_{0}^{2}+\rho\left(1-z_{0}\right)^{2}=k^{2} / \rho$ does not exist when $\gamma \sim 1$, and it is not immediately obvious how to relate the minimum of $z_{n}\left(=m_{n}\right)$ to the subsequent maximum.

We cannot solve (4.8) in closed form, but we only really need the asymptotic form of $\phi$ for large $t$. Clearly $\phi \rightarrow 0$ as $t \rightarrow \infty$, and in fact we may write

$$
\phi-\frac{a}{\omega} \sin [\omega(t-\theta)] \mathrm{e}^{-1 / 2}+O\left(\mathrm{e}^{-3 t / 2}\right)
$$

as $t \rightarrow x$, where

$$
k=\frac{1}{4}+\omega^{2} .
$$

$(4.10)$ is valid for both $\omega^{2}<$ and $>0$. In generai, $a$ and $\theta$ are real $\omega$-dependent coefificients. As $\omega^{2} \rightarrow \theta$, $\phi$ clearly behaves as $a_{0}\left(t-\theta_{0}\right) \mathrm{e}^{-t / 2}$ for large $t$ (but not so large that the term $O\left(\omega^{2}\right)$ in (4.8) is important: that is, $1 \ll t \ll 1 /|\omega|)$; her $a_{0}(>0)$ and $\theta_{0}$ are constants; on the other hand, (4.10) tells us that, for $|\ll t \ll 1 /, \omega|, \phi \approx a(t-\theta) e^{-1 / 2}$; evidently, $a(\omega)=a_{0}+o(1), \theta(\omega)=\theta_{0}+o(1)$ as $\omega \rightarrow 0$, so that we expect $a$ and $\theta$ to be uniformly $O(1)$ functions of $\omega$; in particular, numerical integration shows that

$$
a_{0} \approx 5.404 \ldots, \quad \theta_{0} \approx 3.29 \ldots .
$$


Therefore, as $t \rightarrow \infty$,

$$
\begin{aligned}
x_{0} & \sim-\frac{a}{\sqrt{\rho} \omega}\left[\frac{1}{2} \sin \{\omega(t-\theta)\}-\omega \cos \{\omega(t-\theta)\}\right] e^{-t / 2} \\
& =\frac{\left(-\frac{1}{2}+i \omega\right) a}{2 i \omega \sqrt{\rho}} e^{-i \omega \theta} \exp \left[\left(-\frac{1}{2}+i \omega\right) t\right]+\left(^{*}\right)
\end{aligned}
$$

where $\left(^{*}\right)$ denotes the complex conjugate function. The error in $(4.3)$ is $O(\exp (-3 t / 2))$. As $t \rightarrow \infty$, we also find

$$
z_{0} \sim 2-m_{n}-\frac{1}{2}\left(1-m_{n}\right)\left[\frac{a^{2}}{\omega^{2}} \sin ^{2}\{\omega(t-\theta)\} \mathrm{e}^{-t}+\sigma\left(\mathrm{e}^{-2 t}\right)\right]
$$

It is useful to seek the second-order solutions also. From (4.3) and (4.4), we derive

$$
\frac{d}{d t}\left[y_{0} \cdot-\rho z_{1}\left(1-z_{0}\right)_{1}=-\bar{\gamma} y_{0}^{2}+\rho z_{0}\left(1-z_{0}\right) ;\right.
$$

with the boundary conditions (4.5), this implies

$$
y_{0} y_{1}-\rho z_{1}\left(1-z_{0}\right)=\rho m_{i_{n}}\left(1-m_{n}\right) t+\int_{-\infty}^{t}\left[-\bar{\gamma} y_{0}^{2}+\rho\left\{z_{0}\left(1-z_{0}\right)-m_{n}\left(1-m_{n}\right)\right\}\right] d t .
$$

As $t \rightarrow \infty$, we deduce, after a little algebra,

$$
z_{1} \sim \Gamma\left(\gamma, m_{n}, \rho\right)-\left(2-m_{n}\right) t+\exp [-\mathcal{O}(t)],
$$

where

$$
D\left(\bar{\gamma}, m_{n}, \rho\right)=(1-\bar{\gamma})\left(1-m_{n}\right) \int_{-\infty}^{\infty} \sin ^{2} \phi d t+2\left[\int_{0}^{\infty} \sin ^{2}(\phi / 2) d t-\int_{-\infty}^{0} \cos ^{2}(\phi / 2) d t\right] .
$$

Examination of (4.14) and (4.17) shows that the expansions for $x, y, z$ become invalid when $t \sim 1 / \delta$. Examination of (4.13) suggests a distinguished limit $t \sim 1 / \omega$ when $\omega$ is small (we shall find $\omega \sim \delta^{1 / 3}$ is an appropriate choice in this case); in any case, $z$ has a maximum as $t \rightarrow \infty$ in the fast phase, located approxin. itely at $t \approx \ln (1 / \delta)$. With $\exp [-\sigma(t)] \sim \sigma(\delta)$, we may solve $(d / d t)\left(z_{0}+\delta z_{1}+O\left(\delta^{2}\right)\right)=0$ to find that $z$ is a maximum at $t=t^{*}$, where $t *$ is def ced implicitly by

$$
t^{*}=\ln \left[\frac{\left(1-m_{n}\right) a^{2}}{2\left(2-m_{n}\right) \delta}\right]+\ln \left[\frac{\sin ^{2}\left\{\omega\left(t^{*}-\theta\right)\right\}}{\omega^{2}}-\frac{\sin \left\{2 \omega\left(t^{*}-\theta\right)\right\}}{\omega}\right]+O(\delta) \text {. }
$$

At this value, $z$ has a maximum which we denote by $M_{n}$,

$$
M_{n}=2-m_{n}+\delta\left[D-\left(?-m_{n} \cdot\left\{\left\{+\left[1-\frac{\omega \sin \left\{2 \omega\left(t^{*}-\theta\right)\right\}}{\sin ^{2}\left\{\omega\left(t^{*}-\theta\right)\right\}}\right]^{-1}\right\}\right]+\hat{\sigma}\left(\delta^{2}\right)\right.\right.
$$

The presence of terms $\ln (1 / \delta)$ in $t^{*}$ may mean that $\exp \left(-t^{*}\right) \sim \delta(\delta \ln (1 / \delta))($ if $|\omega|<1)$ so that terms of $O\left(\delta^{2}\right)$ in (4.20) are understood t. include terms such as $O\left(\delta^{2} \ln ^{2}(1 / \delta)\right)$. We also defi.e, for matching purposes, an 'asymptotic' maximum

$$
M_{n}^{\prime}=2-m_{n}+\delta D+O\left(\delta^{2}\right) \text {. }
$$


For large $t(\gg \ln (1 / \delta))$, we may write $(4.14)$ and $(4.17)$ in the form

$$
z-M_{n} \mathrm{e}^{-\delta}+\sigma\left(\delta^{3}\right)
$$

as $t \rightarrow x$.

\subsection{Slow recovery}

As $t \rightarrow x, x$ and $y$ become exponentially small, but crucially non-zero. To all orders of $\delta, z$ then satisfies

$$
\dot{z}=-\delta z+\text { TST. }
$$

so that

$$
z=M_{n} e^{-n !} \text {. }
$$

and $M_{n}^{\prime}$ is givun by (4.21). The exact equation for $x$, with $z$ given by (4.24), to all ordurs of $\delta$ (but neglecting TST), is then

$$
\ddot{x}+(1+\delta \dot{\gamma}) \dot{x}+\left[\rho\left(M_{n}^{\prime} \mathrm{e}^{-\tau}-1\right)+\delta \dot{\gamma}\right] x=0,
$$

where

$$
\tau=\delta t
$$

but still, $\dot{x} \equiv \mathrm{d} x / \mathrm{d} t$. This equation is solvable in an asymptotic manner (Kevorkian and Cole [27]), though some subtlety is involved (Cheng and Wu [23]). However, an exact solution for (4.25) is available. and we use this in preference to an immediate asymptotic solution, as we thereby save some work. The solution to $(4.25)$ is

$$
x=\left[B . Y_{v}\left(\lambda \mathrm{e}^{-1 / 2}\right)+B . J_{1}\left(\lambda \mathrm{e}^{-\mathrm{t} / 2}\right)\right] \exp \left\{-\frac{1}{2}(1+\delta \bar{\gamma}) t\right\}
$$

where $Y$, and $J_{1}$, are Bessel functions,

$$
\lambda=\frac{2}{\delta} \sqrt{\rho M_{n}^{\prime}} . \quad v=\frac{?}{i i}\left[\rho+\frac{1}{4}(1-\delta \bar{\gamma})^{2}\right]^{1 / 2} ;
$$

$B=$ must be determined (with relative errer $O(\delta)$ ) by matching (4.27) to (4.13). Asymptotic behaviours of these Bessel functions when $\lambda \sim \nu \gg 1$ (as here) are given by Abramowitz and Stegun [24]; essentially both functions decay escillatorily if $\lambda^{2} \mathrm{e}^{-\tau}>\nu^{2}(z \geqslant 1+1 / 4 \rho)$, while one grows $\left(Y_{\nu}\right)$ and the other decays $\left(J_{1}\right)$ exponentially if $\lambda^{2} \mathrm{e}^{-\tau}<\nu^{2}(z \leqslant 1+1 / 4 \rho)$ : for $\lambda^{2} \mathrm{e}^{-\tau} \approx \nu^{2}$, there is an Airy-functionlike transition between the two behaviours, with a time scale $t \sim 1 / \delta^{1 / 3}$ : it is this which makes $\omega \sim \delta^{1 / 3}$ an interesting limit in (4.1?). Note that at the beginning of the slow pulse, $z \$ 1+1 / 4 \rho$ depending on $z \approx M_{n}^{\prime} \approx 2-m_{n} \approx 1+k / \rho=1+1 / 4 \rho+\omega^{2} / \rho \equiv 1+1 / 4 \rho$, i.e. $\omega^{2} \leq 0$. This ties in with the behaviour as $t \rightarrow \infty$ of the fast phase, as we should expect.

\subsection{Matching the fast and slow phases}

It will be evident that there are really three different cases, depending on whether $\omega^{2}<0,(1)^{2} \approx 0$, $\omega^{2}>0$, since $(4.27)$ has different asymptotic behaviours at $\tau \approx 0$ for each case. We consider the first two separately (the extension to the third is straightforward). 
(a) $\omega^{2}<0$

Here $M_{n}^{\prime}<1+1 / 4 \rho$, and we may define $\alpha(\tau ; \delta)$ by

$$
\lambda^{2} \mathrm{e}^{-\tau}=\nu^{2} \operatorname{sech}^{2} \alpha, \quad \alpha>0,
$$

that is,

$$
M_{1} e^{--}=\left[1+\frac{1}{4 \rho}(1-\delta \bar{\gamma})^{2}\right] \operatorname{sech}^{2} \alpha(\tau ; \delta) .
$$

with this definition, the asymptotic form of $(4.27)$ for fixed $\alpha(\sim O(1))$ as $\delta \rightarrow 0$ is

$$
x \sim\left[-\frac{B_{+} \exp \{\nu(\alpha-\tanh \alpha)\}}{\sqrt{\frac{1}{2} \pi \nu \tanh \alpha}}\{1+\sigma(\delta)\}+\frac{B_{-} \exp \{-\nu(\alpha-\tanh \alpha)\}}{\sqrt{2 \pi \nu \tanh \alpha}}\{1+\sigma(\delta)\}\right] \exp \left[-\frac{1}{2}(1+\delta \bar{\gamma}) t\right]
$$

To match to $x_{0}$ only, we can ignore terms of relative $O(\delta)$ in (4.31). We define

$$
M_{n}^{\prime}=\left(1+\frac{1}{4 \rho}\right) \operatorname{sech}^{2} \alpha_{0},
$$

and then, with

$$
i \omega=\bar{\omega}=\sqrt{-\omega^{2}}=\frac{1}{2}(1+4 \rho)^{1 / 2} \tanh \alpha_{0}+\sigma(\delta),
$$

we find that as $\tau=\delta t \rightarrow 0,(4.31)$ is

$$
\begin{aligned}
x \sim & -\left(\frac{\delta}{\pi \bar{\omega}}\right)^{1 / 2} B_{+} \exp \left[\frac{1}{\delta}(1+4 \rho)^{1 / 2}\left(\alpha_{0}-\tanh \alpha_{i}\right)+\left(-\frac{1}{2}+\bar{\omega}\right) t-\frac{\bar{\gamma} \alpha_{0}}{(1+4 \rho)^{1 / 2}}+O(\delta)\right] \\
& +\frac{1}{2}\left(\frac{\delta}{\pi \bar{\omega}}\right)^{1 / 2} B_{-} \exp \left[-\frac{1}{\delta}(1+4 \rho)^{1 / 2}\left(\alpha_{0}-\tanh \alpha_{0}\right)+\left(-\frac{1}{2}-\bar{\omega}\right) t+\frac{\bar{\gamma} \alpha_{0}}{(1+4 \rho)^{1 / 2}}+O(\delta)\right],
\end{aligned}
$$

whereas (4.13) implies (to $O(\delta))$

$$
x \sim \frac{\left(-\frac{1}{2}+\bar{\omega}\right) a}{2 \bar{\omega} \sqrt{\rho}} e^{-\bar{\omega} \theta} \exp \left[\left(-\frac{1}{2}+\bar{\omega}\right) t\right]+\frac{\left(-\frac{1}{2}-\bar{\omega}\right) a}{(-2 \bar{\omega}) \sqrt{\rho}} e^{\bar{\omega} \theta} \exp \left[\left(-\frac{1}{2}-\bar{\omega}\right) t\right] .
$$

Matching is therefore determined by prescribing

$$
\begin{aligned}
& \left(\frac{\delta}{\pi \bar{\omega}}\right)^{1 / 2} B_{+} \exp \left[\frac{1}{\delta}(1+4 \rho)^{1 / 2}\left(\alpha_{0}-\tanh \alpha_{0}\right)-\frac{\bar{\gamma} \alpha_{0}}{(1+4 \rho)^{1 / 2}}\right]=\frac{\left(\frac{1}{2}-\overline{0}\right) a}{2 \bar{\omega} \sqrt{\rho}} \mathrm{e}^{-\bar{\omega} \theta}, \\
& \frac{1}{2}\left(\frac{\delta}{\pi \bar{\omega}}\right)^{1 / 2} B_{-} \exp \left[-\frac{1}{\delta}(1+4 \rho)^{1 / 2}\left(\alpha_{0}-\tanh \alpha_{n}\right)+\frac{\bar{\gamma} \alpha_{0}}{(1+4 \rho)^{1 / 2}}\right]=\frac{\left(\frac{1}{2}+\bar{\omega}\right) a}{2 \bar{\omega} \sqrt{\rho}} \mathrm{e}^{\bar{\omega} \theta} .
\end{aligned}
$$

\section{(b) $\omega^{2} \ll 1$}

If $\left|M_{n}^{\prime}-(1+1 / 4 \rho)\right|<1$, then $J_{v}, Y_{v}$ behave initially like Airy functions on a slow time $t \sim 1 / \delta^{1 / 3}$. To isolate this behaviour near $\tau=0$, we define (for $\tau \ll 1$ )

$$
\lambda \mathrm{e}^{-\tau / 2}=\nu+\frac{\nu^{1 / 3}}{2^{1 / 3} \zeta}
$$

Putting also

$$
\tau=\delta^{2 / 3} \tilde{t}, \quad\left(\tilde{t}=\delta^{1 / 3} t\right),
$$


and

$$
\omega^{2}=\delta^{2 / 3} \xi
$$

we find

$$
\zeta=\{2(1+4 \rho)\}^{1 / 3}\left[\frac{2 \xi}{(1+4 \rho)}-\frac{1}{2} \tilde{t}+\frac{\delta^{1 / 3}(\bar{\gamma}+2 \rho D)}{(1+4 \rho)}+O\left(\delta^{2 / 3}\right)\right]
$$

and that

$$
x-\exp \left(-\frac{1}{3} t\right) \cdot\left(\frac{2 \delta}{(1+4 p)^{1 / 2}}\right)^{1 / 3}\left[-B_{+} \operatorname{Bi}(-\zeta)+B-A i(-\zeta)\right]
$$

when $i \sim 1, \delta \rightarrow 0$; relative errors of $O\left(\delta^{2 / 3}\right)$ have been subsumed into (4.40).

The solution (4.40) represents an intermediate limit, which in an appropriate asymptotic analysis would joir the fast phase $t \sim 1$ to the later part of the slow phase $t \sim 1 / \delta$. To match (4.41) to the $t \rightarrow \infty$ limit of the fast phase (4.13). we must the -efore consider a matching region in which $1 \ll t \leqslant 1 / \delta^{1 / 3}$, i.e. $t \gg 1, i \ll 1$. From (4.13), t then follows that

$$
x \sim \frac{a}{\sqrt{\rho}}\left[1+\frac{1}{2} \theta-\frac{1}{2} t+C\left(\delta^{2 / 3} t^{3}\right)\right] \mathrm{e}^{-1 / 2}
$$

To match, we consider an intermediate limit, for example $t=\delta^{1 / 3} t_{\eta} / \eta_{\eta}, t=t_{\eta} / \eta$, and equate terms (multiplying $\left.\exp \left(-\frac{1}{5} t\right)\right)$ of relative orders 1 and $\delta^{1 / 3}$. If we define

$$
\xi^{*}=\left\{; \frac{4}{+4 \rho}\right\}^{2 / 3}\left[\xi+\frac{\delta^{1 / 3}(\bar{\gamma}+2 \rho D)}{2}\right]
$$

the, $B$, are given to relative $O\left(\delta^{2 / 3}\right)$ by

$$
\left(\begin{array}{cc}
-\mathrm{Bi}\left(-\zeta^{*}\right) & \mathrm{Ai}\left(-\zeta^{*}\right) \\
-\mathrm{Bi}^{\prime}\left(-\zeta^{*}\right) & \mathrm{Ai}^{\prime}\left(-\zeta^{*}\right)
\end{array}\right)\left(\begin{array}{l}
\mathrm{B}_{-} \\
\mathrm{B}_{-}
\end{array}\right)=\left(\begin{array}{c}
\frac{(1+4 \rho)^{1 / 6}}{2^{1 / 3} \delta^{1 / 3}} \frac{\left(1+\frac{1}{2} \theta\right) a}{\sqrt{\rho}} \\
-\frac{a}{2^{2 / 3} \delta^{2 / 3}} \frac{1}{\sqrt{\rho}(1+4 \rho)^{1 / 6}}
\end{array}\right)
$$

and since $a=a(\omega), \theta=\theta(\omega)$, we require $a$ and $\theta$ in (4.44) correct to $O\left(\delta^{1 / 3}\right)$, i.e. $O(\omega)$. The Wronskian of $\mathrm{Ai}$ and $\mathrm{Bi}$ is $\pi^{-1}$, so

$$
\left(\begin{array}{l}
B_{+} \\
B_{-}
\end{array}\right)=\frac{\pi a}{\sqrt{\rho} \delta^{2 / 3}}\left(\begin{array}{ll}
\mathrm{Ai}^{\prime}\left(-\zeta^{*}\right) & -\mathrm{Ai}\left(-\zeta^{*}\right) \\
\mathrm{Bi}^{\prime}\left(-\zeta^{*}\right) & -\mathrm{Bi}\left(-\zeta^{*}\right)
\end{array}\right)\left(\begin{array}{c}
\frac{\delta^{1 / 3}}{2^{1 / 3}}(1+4 \rho)^{1 / 6}\left(1+\frac{1}{2} \theta\right) \\
-\frac{1}{2^{2 \pi /}(1+4 \rho)^{\pi / 6}}
\end{array}\right)
$$

particularly,

$$
B .=\frac{\pi a}{\sqrt{\rho} \delta^{2 / 3} 2^{2 / 3}(1+4 \rho)^{1 / 6}}\left[\mathrm{Ai}\left(-\zeta^{*}\right)+2^{1 / 3} \delta^{1 / 3}(1+4 \rho)^{1 / 3}\left(1+\frac{1}{2} \theta\right) \mathrm{Ai}^{\prime}\left(-\zeta^{*}\right)\right],
$$

with relative error $O\left(\delta^{2 / 3}\right)$; equivalently

$$
B_{+}=\frac{\pi a}{\sqrt{\rho} \delta^{2 / 3} 2^{2 / 3}(1+4 \rho)^{1 / 6}}\left\{\mathrm{Ai}\left(-\bar{\zeta}+O\left(\delta^{2 / 3}\right)\right)\right\}
$$


where

$$
\bar{\zeta}=\zeta^{*}-2^{1 / 3} \delta^{1 / 3}(1+4 p)^{1 / 3}\left(1+\frac{1}{2} \theta\right) .
$$

Evidently, we require a to $O\left(\delta^{1 / 3}\right)$, but $\theta$ only to $O(1)$. In terms of $M_{n}^{\prime}$, we have

$$
M_{n}^{\prime}=1+\frac{1}{4 \rho}+\frac{\omega^{2}}{\rho}+\delta D=1+\frac{1}{4 \rho}+\frac{\delta^{2 / 3} \xi}{\rho}+\delta D,
$$

whence (4.43) and (4.47) give

$$
M_{n}^{\prime}=1+\frac{1}{4 \rho}+\frac{\delta^{2 / 3}(1+4 \rho)^{2 / 3}}{2^{4 / 3} \rho} \bar{\zeta}+\frac{\delta}{2 \rho}\left[(1+4 \rho)\left(1+\frac{1}{2} \theta\right)-\bar{\gamma}\right]
$$

(c) $\omega^{2}>0$

This case is easily studied in a similar manner, but we omit the relevant analysis here.

\subsection{Matching to the next pulse}

As $\tau$ increases through the slow phase, $\alpha$ defined by (4.29) increases, and thus so also does $\alpha-\tanh \alpha$; hence $J_{v}$ in (4.27) continues (for $\tau>0$ ) to decay towaris zero. $Y_{v}$, on the other hand, first decreases exponertially, but eventually (when $\nu(\alpha-\tanh \alpha)-t / 2>0$ ) starts to increase again. Thus eventually $x$ becomes (rapidly) $O(1)$ and a new pulse begins. To determine when this occurs, we define

$$
\tau=\tau^{*}+\delta t^{\prime},
$$

and will determine conditions on $\tau^{*}$ so that $t^{\prime}=0$ is the 'location' of the next fast pulse: specifically so that as $t^{\prime} \rightarrow-\infty$, the analogue of (4.6) and (4.9) holds. Identical considerations hold if $\left.M_{n}^{\prime}-\mid 1+1 / 4 \rho\right) \mid \ll$ 1 (or if $\left.M_{n}^{\prime}>1+1 / 4 \rho\right) ; x$ does not grow until the asympiotic regime (4.31) is entered, and $\alpha$ is large enough.

From (4.24),

$$
z \sim M_{n}^{\prime} \mathrm{e}^{-\tau}
$$

with transcendentally small error. (4.5) then implies that we define the next value of $m_{n}$, i.e. $m_{n+1}$, as

$$
m_{n+1}=M_{n}^{\prime} \mathrm{e}^{-r^{*}} \text {. }
$$

Define $\alpha^{*}$ by

$$
m_{n+1}=M_{n}^{\prime} \mathrm{e}^{-\tau^{*}}=\left(1+\frac{1}{4 \rho}\right) \operatorname{sech}^{2} \alpha^{*}
$$

then, with

$$
k^{\prime}=\rho\left(1-m_{n+1}\right)=-\frac{1}{4}+\omega^{* 2} \quad\left(\omega^{*}>\frac{1}{2}\right),
$$

we find

$$
\omega^{*}=\frac{1}{2}(1+4 \rho)^{1 / 2} \tanh \alpha^{*},
$$

and our requirement on $x$ as $\mathfrak{l}^{\prime} \rightarrow-\infty$ is, from (4.9)

$$
x \sim \mp \frac{\left(-\frac{1}{2}+\omega^{*}\right)}{\sqrt{\rho}} \exp \left[\left(-\frac{1}{2}+\omega^{*}\right) t^{\prime}\right]
$$


to $O(\delta)$. Here, a plus sign indicates a change of sign of $x$ (and $y$ ) in the next pulse compared to the previou; pulse. Expanding (4.31) for small $\delta t^{\prime}$, and retaining only the relevant $B_{+}$term (the other is TST). we find

$$
\begin{aligned}
x & \sim \frac{-B}{\sqrt{\Sigma} \frac{T}{\pi v \tanh \alpha^{*}}}[1+C(\delta)] \exp \left[\nu(\alpha-\tanh \alpha)-\frac{1}{2}(1+\delta \bar{\gamma})\left(\frac{\tau^{*}}{\delta}+t^{\prime}\right)\right] \\
& -B \cdot\left(\frac{\delta}{\pi \omega^{*}}\right)^{1 / 2} \exp \left[\frac{1}{\delta}\left\{(1+4 \rho)^{1 / 2}\left(\alpha^{*}-\tanh \alpha^{*}\right)-\frac{1}{2} \tau^{*}\right\}\right. \\
& \left.-\bar{y}\left\{\frac{\alpha^{*}}{(1+4 \rho)^{1 / 2}}+\frac{1}{2} \tau^{*}\right\}\right] \exp \left[\left(-\frac{1}{2}+\omega^{*}\right) t^{\prime}\right]
\end{aligned}
$$

the sign of $B_{+}$determines whether there is a reversal of $x$ (and $y$ ): if $B_{+}<0$, there is a reversal, whereas if $B_{+}>0$, there is not. Matching of (4.55) and (4.56) then determines $\tau^{*}$ by

$$
\frac{\omega^{*}-!}{\sqrt{\rho}}=\left|B_{+}\right|\left(\frac{\delta}{\pi \omega^{*}}\right)^{1 / 2} \exp \left[\frac{1}{\delta}\left\{(1+4 \rho)^{1 / 2}\left(\alpha^{*}-\tanh \alpha^{*}\right)-\frac{1}{2} \tau^{*}\right\}-\bar{\gamma}\left\{\frac{\alpha^{*}}{(1+4 \rho)^{1 / 2}}+\frac{1}{2} \tau^{*}\right\}+O(\delta)\right] \text {. }
$$

\subsection{Reduction to a difference equation} is

We are now in a pesition to form a difference equation for $M_{n}$. In terms of $M_{n}^{\prime}$, the $n$th maximum

$$
M_{n}^{\prime}\left[1-\delta\left\{t^{*}+\left[1-\frac{\omega \sin \left\{2 \omega\left(t^{*}-\theta\right)\right\}}{\sin ^{2}\left\{\omega\left(!^{*}-\theta\right)\right\}}\right]^{-1}\right\}+\sigma\left(\delta^{2}\right)\right]
$$

here. $t^{*}$ is the solution of (4.19) where $a, \theta$ are determined from (4.8) and (4.10), and we may take $n_{n} \approx 2-M_{n}$. Briefly.

$$
M_{n}=M_{n}^{\prime}\left[1-\delta \chi\left(2-M_{n}^{\prime} \rho\right)+C\left(\delta^{\prime}\right)\right] \text {. }
$$

For such an $M_{n}, M_{n+1}$ is then determined from (4.20) and (4.51) as

$$
M_{n+1}=\left[2-M_{n}^{\prime} \mathrm{e}^{-*}\right]\left[1-\delta \chi\left(M_{n}^{\prime} \mathrm{e}^{-+^{*}}, \mu^{\prime}\right)\right]+\delta D\left(\bar{\gamma}, M_{n}^{\prime} \mathrm{e}^{-\tau^{*}}, \rho\right)+O\left(\delta^{2}\right) ;
$$

here. $D$ is given by (4.18). Recall that $\chi$ is $O, \ln (1 / \delta))$, and then $O\left(\delta^{2}\right)$ is really $O\left(\delta^{2} \ln ^{2}(1 / \delta)\right)$. The quantity $\tau^{*}$ in $(4.60)$ is determined implicitly from $(4.57)$, that is

$$
\tau^{*}=2(1+4 \rho)^{1 / 2}\left(\alpha^{*}-\tanh \alpha^{*}\right)-\delta \bar{\gamma}\left\{\frac{2 \alpha^{*}}{(1+4 \rho)} T^{T / 2}+\tau^{*}\right\}+2 \delta \ln \left[\left(\frac{\delta}{\pi \omega^{*}}\right)^{1 / 2} \frac{\sqrt{\rho}}{\left(\omega^{*}-\frac{1}{2}\right)}\left|B_{+}\right|\right]+O\left(\delta^{2}\right),
$$

and the additional quantities $\omega^{*},\left|B_{+}\right|$, and $\alpha^{*}$ are given by

$$
\begin{aligned}
& \omega^{*}=!(1+4 \rho)^{1 / 2} \tanh \alpha^{*} \\
& M_{n}^{\prime} \mathrm{e}^{* *}=\left(1+\frac{1}{4 \rho}\right) \operatorname{sech}^{2} \alpha^{*}
\end{aligned}
$$

and $B_{+}$is given by (4.32), (4.33) and (4.36) if $M_{n}<1+1 / 4 \rho$ (note $B_{+}>0$ ), and by (4.46) and (4.48) if $\left.\mid M_{n}-1+1 / 4 \rho\right) \mid \ll 1$. 


\section{Structure of the difference equation}

We have given quite a complicated description of the difference equation, in order to try and keep track of the errors which arise. For most purposes, of course, one can simply take the leading order form of the expressions, and in this case it becomes much simpler to visualise the graph of $M_{n+1}$ versus $M_{n}$. From the equations at the end of section 4 , we have

$$
\begin{aligned}
& M_{n+1}=2-M_{n} e^{-\tau^{*}}+O(\delta), \\
& M_{n} e^{-\tau^{*}}=\left(1+\frac{1}{4 \rho}\right) \operatorname{sech}^{2} \alpha^{*}+O(\delta),
\end{aligned}
$$

if $M_{n}<1+1 / 4 \rho$, then

$$
\begin{aligned}
& M_{n}=\left(1+\frac{1}{4 \rho}\right) \operatorname{sech}^{2} \alpha_{0}+O(\delta), \\
& \tau^{*}=2(1+4 \rho)^{1 / 2}\left[\left(\alpha^{*}-\tanh \alpha^{*}\right)-\left(\alpha_{0}-\tanh \alpha_{0}\right)\right]+O(\delta),
\end{aligned}
$$

whereas if $\left|M_{n}-(1+1 / 4 \rho)\right| \leqslant 1$, then

$$
\begin{aligned}
& M_{n}=1+\frac{1}{4 \rho}+\frac{\delta^{2 / 3}(1+4 \rho)^{2 / 3}}{2^{4 / 3} \rho} \bar{\zeta}+O(\delta), \\
& \tau^{*}=2(1+4 \rho)^{1 / 2}\left(\alpha^{*}-\tanh \alpha^{*}\right)+2 \delta \ln |\mathrm{Ai}(-\bar{\zeta})|+O(\delta),
\end{aligned}
$$

and if $\left|M_{n}-(1+1 / 4 \rho)\right| \ll 1$, then $O(\delta)$ is realiy $O(\delta \ln (1 / \delta))$. We retain the term $2 \delta \ln |\operatorname{Ai}(-\bar{\zeta})|$, since it is not uniformly small near zeros of Ai. All other $O(\delta)$ terms are uniformly relatively small, and may as a first approximation be ignored. Nevertheless, $\delta$ is essentially present when $M_{n} \approx 1+1 / 4 \rho$, and a similar conclusion is valid when $M_{n}>1+1 / 4 \rho$.

\subsection{Form of the envelope difference equation}

We mean to determine the form of the difference equation give 1 by (5.1)-(5.4), and in particular we shall show that Lorenz' cusp is due to the term $2 \delta \ln |\mathrm{Ai}(-\bar{\zeta})|$ in ( $₫ .4$ ). The cusp (in fact, a number of them) occur at values of $M_{n}$ where $\bar{\zeta}$ approaches a zero of $\mathrm{Ai}(-\bar{\zeta}$, : away from such zeros, however, this term is small, and may be neglected consistently with other erms of $O(\delta)$ : we are thus led to consider the envelope difference equation, which is determined by $(: .1)-(5.3)$ together with

$$
\tau^{*}=2(1+4 \rho)^{1 / 2}\left(\alpha^{*}-\tanh \alpha^{*}\right), \text { if } M_{n}>1+1 / 4 \rho
$$

(strictly, we have only derived this for $M_{n} \approx 1+1 / 4 p$, but the same $\mathrm{fc} \mathrm{rm}$ is valid at higher $M_{n}$ ). We will show that the difference equation (5.1)-(5.4) may be viewed as being $g$ ven by the envelope form, with a number of isolated cusps superimposed on it.

To examine the shape of the envelope curve, it is convenient to $u$ 'rite

$$
M_{n}=1+\theta, \quad M_{n+1}=1+\phi,
$$

and compute the derivative $\phi^{\prime}(\theta)$. In $(\theta, \phi)$ coordinates (beware that $\theta$ here is unrelated to the phase $\theta$ in section 4, which later will play no further part in this analysis), the origin $(0,0)$ corresponds to $M_{n}=M_{n+1}=1$, i.e. (either) non-trivial steady state of the Lorenz system. After some algebra, we find 
from $(5.1)-(5.3)$ and $(5.5)$ that

$$
\frac{d \phi}{d \theta}= \begin{cases}\frac{(1-\phi)\left[1-\{1-4 \rho \theta\}^{1 / 2}\right]}{(1+\theta)\left[\{1+4 \rho \phi\}^{1 / 2}-1\right]}, & \theta<1 / 4 \rho, \\ \frac{(1-\phi)}{(1+\theta)\left[\{1+4 \rho \phi\}^{1 / 2}-1\right]}, & \theta<1 / 4 \rho,\end{cases}
$$

with $\phi(0)=0$ and $\phi$ continuous at $\theta=1 / 4 \rho$.

Two applications of L'Hôpital's rule show that

$$
\phi^{\prime}(0)=1, \quad \phi^{\prime \prime}(0)=\frac{4}{3}(\rho-1) ;
$$

it follows from this that the fixed point $M_{n}=1$ becomes unstable as $\rho$ increases through 1: see fig. 4 for the relevant diagram. This agrees with the linear instability criterion $r_{c}=\sigma(\sigma+b+3) /(\sigma-b-1)$, which implies the critical value of $\rho$ is $\rho_{\mathrm{c}}=1+O(\delta)$ as $\delta \rightarrow 0$. From (5.7) and (5.8) we can deduce $\phi$ increases monotonically from 0 when $\theta=0$ to 1 as $\theta \rightarrow \infty$. A schematic form of $\phi(\theta)$ is then shown in fig. 5 (numerical plots bear this out, and indeed the concavity for $\theta<1 / 4 \rho$ is suggested by these plots). We call the curve generated by (5.7), with $\phi(0)=0$ and $\phi$ continuous at $1 / 4 \rho$, the envelope differerence equation.

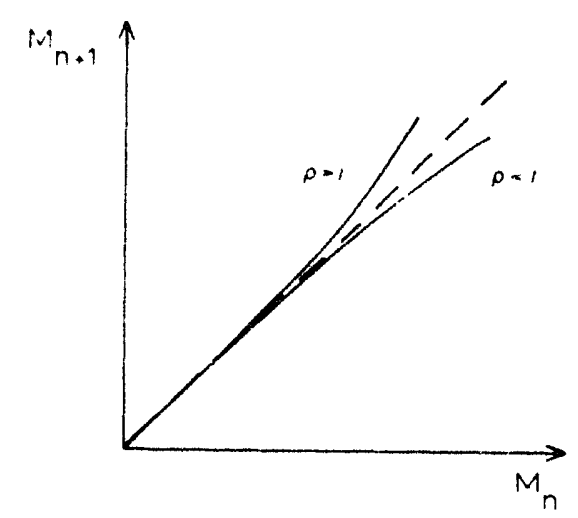

Fig. 4. Bifurcation sequence of the fixed pont $M_{n}=M_{n+1}=1$ in the Lorenz equations as $\sigma \rightarrow \infty, r / \sigma$ finite. The critical value of $r$ is given by $r / \sigma=\rho=1+C(1 / \sigma)$.

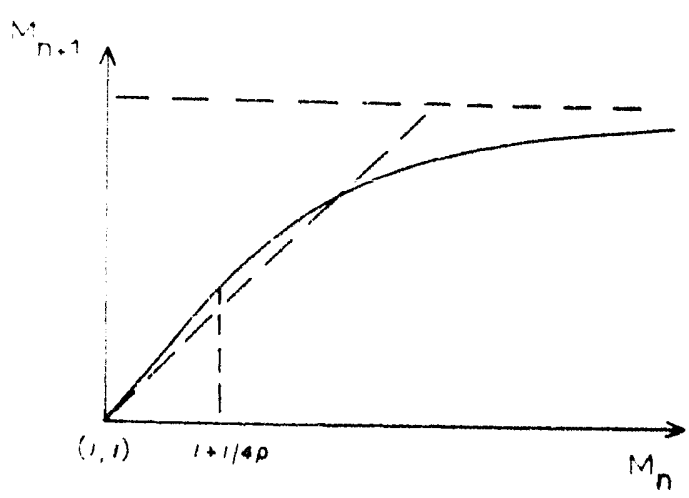

Fig. 5. Schematic form of the envelop' difference equation $M_{n+1}\left(M_{n}\right) \cdot d^{2} M_{n+1} / d M_{n}^{2}$ is discontinuous at $M_{n}=1+1 / 4 p$. 


\subsection{Difference equation with cusps}

We now return to the full difference equation, (5.1)-(5.4). For $M_{n}<1+1 / 4 p$, the envelope equation is relevant. For $M_{n}>1+1 / 4 p$, however, the extra term in (5.4) needs to be considered. Specifically, when $M_{n}=1+1 / 4 p$, we have $($ to $\sigma(\delta \ln (1 / \delta)))$

$$
\begin{aligned}
& M_{n+1}=2-M_{n} e^{-r^{*}}, \\
& M_{n} e^{-\tau^{*}}=\left(1+\frac{1}{4 \rho}\right) \operatorname{sech}^{2} \alpha^{*}, \\
& M_{n}=1+\frac{1}{4 \rho}+\frac{\delta^{2 / 3}(1+4 \rho)^{2 / 3}}{2^{4 / 3} \rho} \zeta, \\
& \tau^{*}=2(1+4 \rho)^{1 / 2}\left(\alpha^{*}-\tanh \alpha^{*}\right)+2 \delta \ln |\operatorname{Ai}(-\zeta)|
\end{aligned}
$$

The graph of $\mathrm{Ai}(-\zeta)$ is shown in fig. 6: it has zeros at the sequence of positive values $\zeta_{1} \approx 2.34$, $\zeta_{2} \approx 4.09, \zeta_{3} \approx 5.52, \ldots$; provided $\left|\zeta-\zeta_{1}\right|>0$, then the envelope approximation to the difference equation given above is valid - this is true also for $M_{n}>1+1 / 4 p$. Thus the correction is only important when $\left|\zeta-\zeta_{i}\right| \leftarrow 1$ for some $i$. In this case $\mathrm{Ai}(-\zeta) \approx-\mathrm{Ai}^{\prime}\left(-\zeta_{i}\right)\left(\zeta-\zeta_{i}\right)$, and (neglecting $O(\delta)$ ), (5.9)4 gives

$$
\tau^{*}=2(1+4 \rho)^{1 / 2}\left(\alpha^{*}-\tanh \alpha^{*}\right)+28 \ln \left|\zeta-\zeta_{i}\right| \text {. }
$$

$(5.9)_{2}$ gives

$$
\begin{aligned}
& \mathrm{e}^{-\tau^{*}}=\left[1+\mathcal{O}\left(\delta^{2 / 3}\right)\right]^{-1} \operatorname{sech}^{2} \alpha^{*}, \\
& \tau^{*} \approx 2 \ln \cosh \alpha^{*},
\end{aligned}
$$

so that $(5.10)$ is

$$
\delta \ln \left[\frac{1}{\left|\zeta-\zeta_{1}\right|}\right]=(1+4 \rho)^{1 / 2}\left(\alpha^{*}-\tanh \alpha^{*}\right)-\ln \cosh \alpha^{*}
$$

Now as $\zeta \rightarrow \zeta_{1}$, we see that $\alpha^{*} \rightarrow \infty$, and hence $\tau^{*} \rightarrow \infty$ also; for these values of $M_{n}$, the interval between

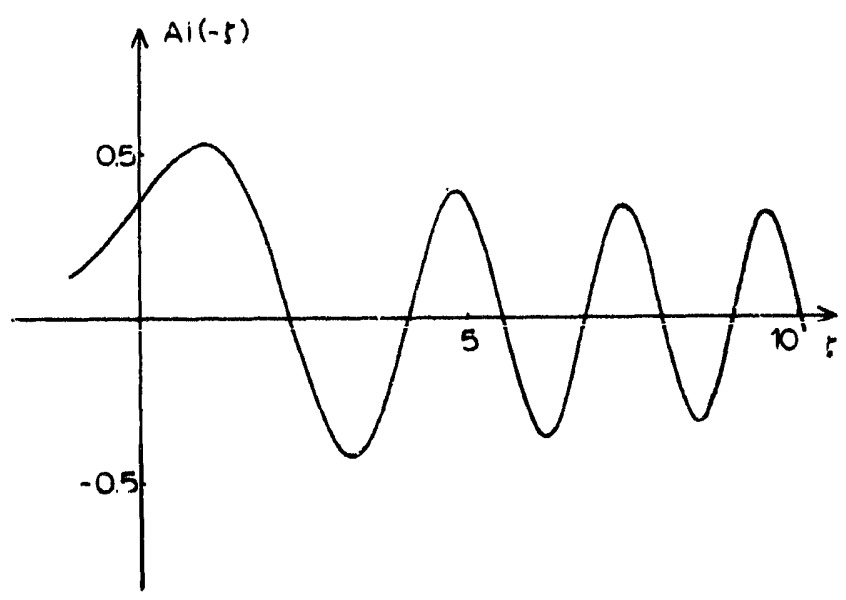

Fig. 6. Graph of Ai(- $\emptyset$ versus :. Altlough schematic, this is fairly accurately copied from the corresponding figure in Abramowitz and Stegun [24]. 
pulses increases indefinitely, and the values of $M_{n} \exp \left(-\tau^{*}\right)$ decreases towards zero. As noted by Lorenz [3]. this means that these values of $M_{n}$ are followed by very close approaches to the origin.

Now when $\alpha * \$ 1,(5.12)$ is approximately

$$
\delta \ln \left|\frac{1}{\zeta-\zeta_{i}}\right|=\left[(1+4 \rho)^{1 / 2}-1\right] \alpha^{*}-\left[(1+4 \rho)^{1 / 2}-\ln 2\right] ;
$$

from (5.11).

$$
广^{*} \approx 2 \alpha^{*}-2 \ln 2 \text {. }
$$

thus

$$
\begin{aligned}
M_{n-1} & \approx 2-\left(1+\frac{1}{4 \rho}\right) \cdot 4 \exp \left(-2 \alpha^{*}\right) \\
& \approx 2-4\left(1+\frac{1}{4 \rho}\right) \exp \left[-\frac{2}{\left\{(1+4 \rho)^{1 / 2}-1\right\}}\left\{(1+4 \rho)^{1 / 2}-\ln 2+\delta \ln \left|\frac{1}{\zeta-\zeta_{i}}\right|\right\}\right]
\end{aligned}
$$

We define

$$
\begin{aligned}
& \beta=\frac{2 \delta}{\left\{(1+4 \rho)^{1 / 2}-1\right\}} \\
& k=4\left(1+\frac{1}{4 \rho}\right) \exp \left[\frac{-2\left\{(1+4 \rho)^{1 / 2}-\ln 2\right\}}{\left\{(1+4 \rho)^{1 / 2}-1\right\}}\right]
\end{aligned}
$$

then the difference equation when $\zeta \approx \zeta_{i}$ is given for each $i$, by

$$
M_{n-1}=2-k\left|\zeta-\zeta_{i}\right|^{\beta} \text {. }
$$

which has the appearance of a cusp. Away from the zeros $\zeta_{i}$, the difference equation is appruximately given hy the envelope form and thus a composite schematic form of the difference equation may be represented as in fig. 7 .

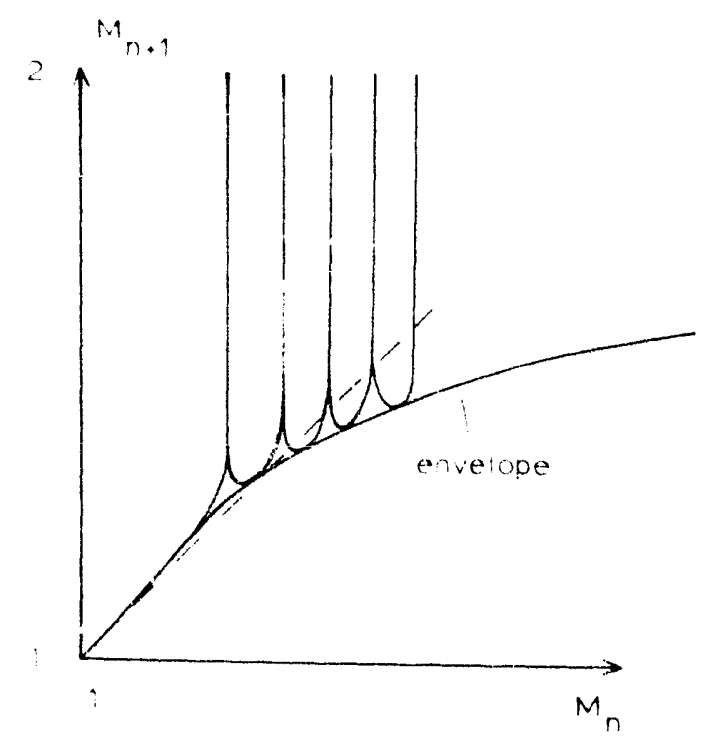

Fig. 7. Schematic form of the difference equation with cusps. 
6. Predictions of the analysis, and comparison with numerical experiments

At the end of section 5 , we have obtained a difference equation which has cusps as shown in fig. 7. Before comparing this predicted result with numerical results, let us derive one further form of the equation.

\subsection{Uniform approximation}

In the cusps, we have shown $\alpha^{*} \gg 1$. Let. us see how far we can extend this result: that is, let us expand (5.9) for $\alpha^{*}$, and hence also $\tau^{*}$, large. Following the procedure in section 5 , we have (neglecting $O(\delta \ln \delta)$ )

$\tau^{*}=2(1+4 \rho)^{1 / 2}\left(\alpha^{*}-1\right)+2 \delta \ln |\mathrm{Ai}(-\zeta)|$,

with an error $\sim \exp \left(-2 \alpha^{*}\right)$,

$M_{n} \mathrm{e}^{-\tau^{*}}=4\left(1+\frac{1}{4 \rho}\right) \mathrm{e}^{-2 a^{*}}$,

with relative error $\exp \left(-2 \alpha^{*}\right)$, and thus

$$
\begin{aligned}
\tau^{*}- & \ln M_{n}=2 \alpha^{*}-\ln \left[4+\frac{1}{\rho}\right] \\
\Rightarrow & 2(1+4 \rho)^{1 / 2}\left(\alpha^{*}-1\right)+2 \delta \ln |\mathrm{Ai}(-\zeta)| \\
= & 2 \alpha^{*}+\ln \left[\frac{M_{n}}{1+1 / 4 \rho}\right]-2 \ln 2,
\end{aligned}
$$

and so

$$
\begin{aligned}
2 \alpha^{*}= & \frac{1}{\left[(1+4 \rho)^{1 / 2}-1\right]}\left[2(1+4 \rho)^{1 / 2}-2 \ln 2\right. \\
& \left.+\ln \left[\frac{M_{n}}{1+1 / 4 \rho}\right]-2 \delta \ln |\mathrm{Ai}(-\zeta)|\right]
\end{aligned}
$$

The error in (6.3) is $\sim \exp \left(-2 \alpha^{*}\right)$, and thus not asymptotically small (except $n$ zar.zeros of $\mathbf{A i}$ ); nevertheless, it giv is a reasonable idea of the shape of the difference equation, and gives the basis for a more accurate analytic determination of the curve. For $1<\rho<2, \exp \left(-2 \alpha^{*}\right) \approx 0.1$.
Substituting (6.3) into (6.2), we find that the difference equation (5.9), is

$$
M_{n+1}=2-k\left[\frac{M_{r}}{(1+1 / 4 \rho)}\right]^{-1 /(1+4 \rho)^{1 / 2-1\}}}|\mathrm{Ai}(-\zeta)|^{\beta},
$$

where $\zeta, k, \beta$ are defined in (5.9), (5.16) and (5.17), respectively. For $|\zeta| \leqslant 1, \quad(6.4)$ is, parametrically,

$$
\begin{aligned}
& M_{n}=\left(1+\frac{1}{4 \rho}\right)\left[1+\frac{\delta^{2 / 3} 2^{2 / 3} \zeta}{(1+4 \rho)^{1 / 3}}\right], \\
& M_{n+1}=2-k \mid \operatorname{Ai}(-\zeta)^{\beta} \exp \left[-\lambda \delta^{2 / 3} \zeta\right],
\end{aligned}
$$

and the parameters are

$$
\begin{aligned}
& k=4\left(1+\frac{1}{4 \rho}\right) \exp \left[\frac{-2\left\{(1+4 \rho)^{1 / 2}-\ln 2\right\}}{\left\{(1+4 \rho)^{1 / 2}-1\right\}}\right], \\
& \beta=\frac{2 \delta}{\left\{(1+4 \rho)^{1 / 2}-1\right\}} \\
& \lambda=\frac{2^{2 / 3}}{(1+4 \rho)^{1 / 3}\left\{(1+4 \rho)^{1 / 2}-1\right\}}
\end{aligned}
$$

The form of (6.4) or (6.5) is as shown in fig. 7 for $M_{n} \geqslant 1+1 / 4 \rho$. Belo $\because$ this $\alpha^{*}$ approaches zero as $M_{n} \rightarrow 1$, and the form of (6.4) and (6.5) is untenable.

\subsection{Numerical results}

The prediction of many cusps was suggested by a numerical computation which appeared to show two. This prediction was subsequently verified by plots of successive maxima using multiple starting values which indicate the presence of at least four cusps in the difference equation. Four such plois are shown in fig. 8 for different values of $r$ at $\sigma=50, b=1$, that is, $\delta=0.02$. Discrepancies $b$-tween the height of these cusps and those shown in fig. 7 are discussed in 6.5 below. In each of the first three cases the system evolved into a limit cycle, corresponding to a fixed point of the difference 

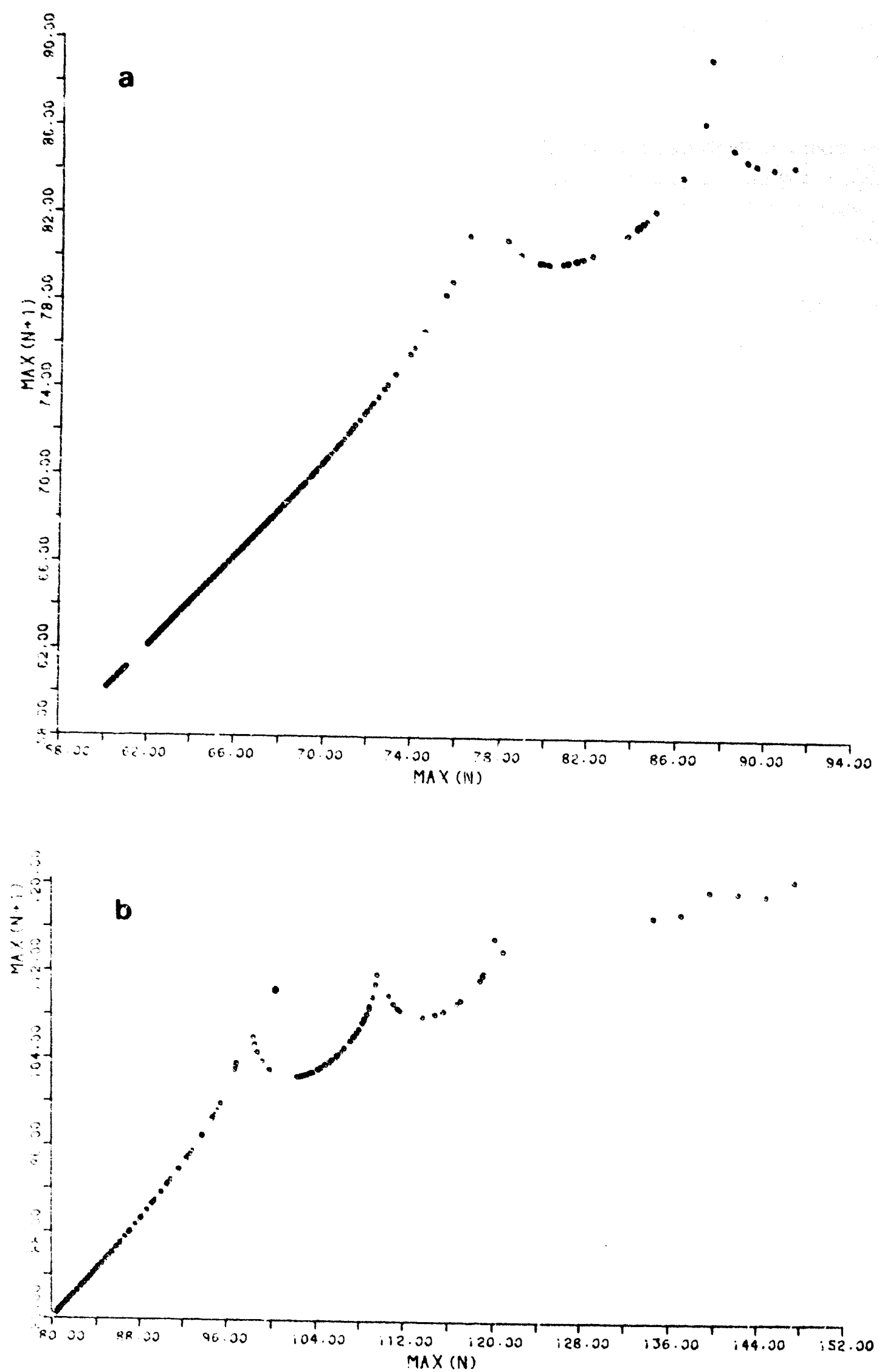

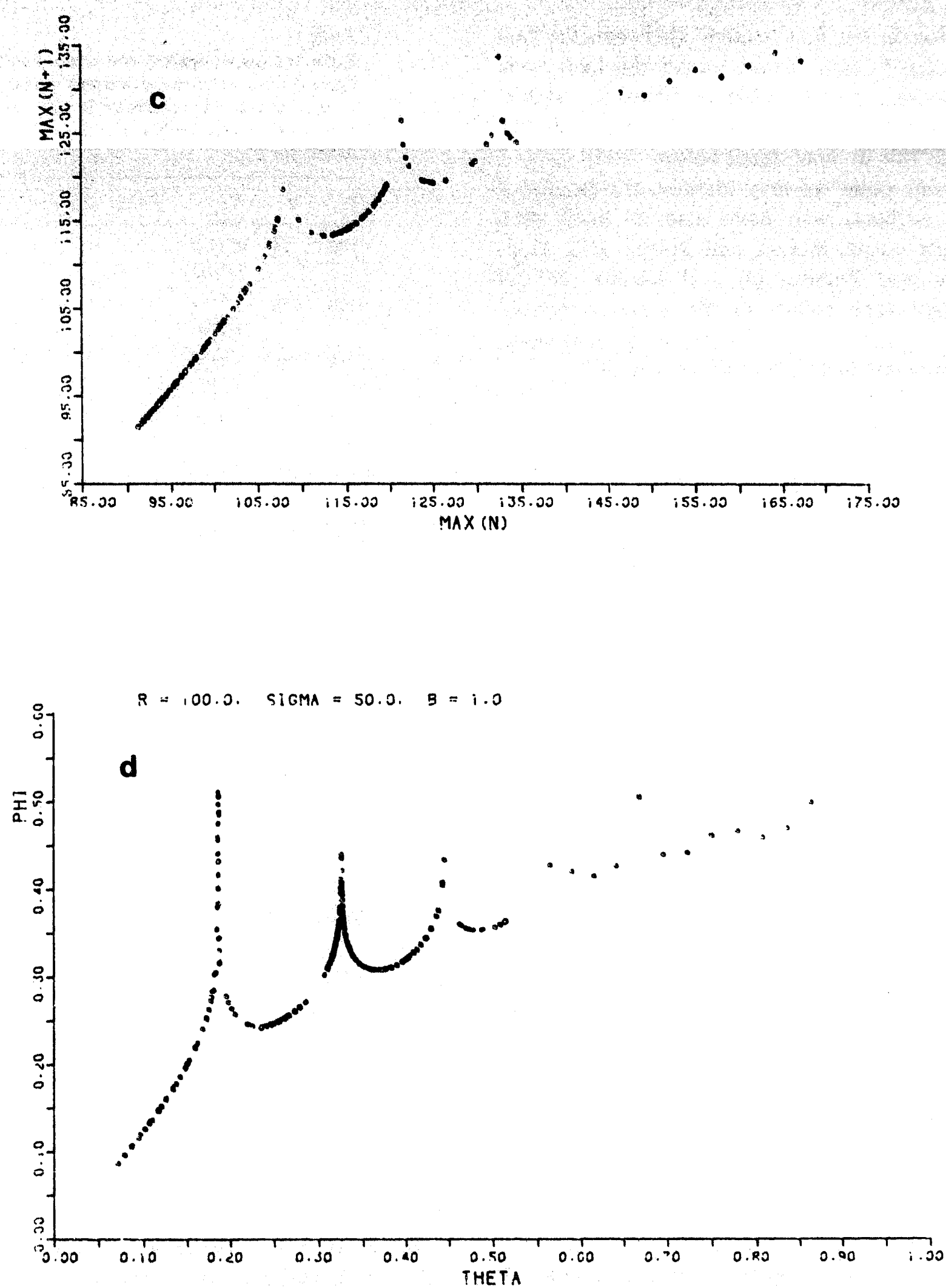

Fig. 8. Plots of the difference equation obtained by numerically solving the Lorenz equations at values $b=1, \sigma=50$ and $(a) r=60$; (b) $r=80$; (c) $r=90$; (d) $r=100$. See text for further explanation. A special 'cusp-sharpening' routine was used to get further up the cusps in (d). 
equation in the first 'trough' (between the first and second cusps). In the fourth, this limit cycle still exists, but in addition, there is 'stable' aperiodic behaviour in the second trough: we discuss this in more detail below.

At this point we may mention the results of other authors. who have also obtained such multiple cusps. Marzec and Spiegel [25], Man. neville and Pomeau [4] and Lorenz [26] all obtained such results in the same or related models. Lorenz' results were for decreasing $b$; we comment further on this in section 7 .

\subsection{Position of cusps}

The zeros $a_{s}$ of $\mathrm{Ai}(z)$ are given by

$a_{1}=-2.33810 \ldots$

$a_{2}=-4.08794 \ldots$

$a_{3}=-5.52055 \ldots$

$a_{4}=-6.79670 \ldots$

The zeros of $\mathrm{Ai}(-\zeta)$ are jusi given by $\zeta=\left|a_{s}\right|$. Therefore we predict from (6.5) that the cusps of th: ditierence curve will be located approximately at the values

$M_{n}=C_{s}=1+\frac{1}{4 \rho}+\frac{\delta^{3 / 2}(1+4 \rho)^{2 / 3}}{2^{4 / 3} \rho}\left|a_{s}\right|$.

in table I we show some predicted cusp values $C$, compared with the actual locations determined from a numerical solution of the uriginal equations. We hope it is evident from this table that the predicted and computed values agree reasonably with each other, becoming more accurate as $\sigma$ increases.

On the other hand, the agreement is not so good that the analysis can be said to be thoroughiy quantitatively endorsed. In fact, the first version of this paper computed predicted values of $C_{s}$ using (6.8) with $\left|a_{s}^{\prime}\right|$ (i.e. zeros of $\mathrm{Ai}^{\prime}(-\zeta)$ ) rather than $\left|a_{s}\right|$ (this was due to imprecise reasoning concerning (4.10) when $\omega$ is
Table I

Predicted (upper figures) and actual (lower figures) cusp locations at various $r$ and $\sigma$ values, and $b=1$. Al:curacy in the computed values are 0.0001 for $C_{1}$ and $C_{2}$, 0.0002 for $C_{3}$.

\begin{tabular}{lllll}
\hline$r$ & $\sigma$ & $C_{1}$ & $C_{2}$ & $C_{3}$ \\
\hline 60 & 50 & 1.3922 & & \\
& & 1.2862 & & \\
100 & 50 & 1.2729 & & \\
& & 1.1860 & & \\
116 & 100 & 1.3332 & & \\
& & 1.2703 & & \\
320 & 300 & 1.2931 & 1.3371 & 1.3731 \\
& & 1.2661 & 1.3136 & 1.3536 \\
360 & 300 & 1.2640 & 1.3057 & 1.3398 \\
& & 1.2382 & 1.2832 & 1.3212 \\
550 & 500 & 1.2685 & 1.2993 & 1.3246 \\
& & 1.2509 & 1.2835 & 1.3109 \\
600 & 500 & 1.2480 & & \\
& & 1.2309 & & \\
\hline
\end{tabular}

small); however, the resulting predictions were much better than the values in table I! Our feeling is that this may be fortuitous, but in any case one need not be unduly worried about the values in table $I$, since their intended accuracy is only to $O(\delta \ln (1 / \delta))$, whereas the cusp spacing itself is only $O\left(\delta^{2 / 3}\right)$ : these are numerically comparable for moderately small values of $\delta$; for example, if $\sigma=1000, b=1$, then $\delta^{2 / 3}=0.01$, $\delta \ln (1 / \delta)=0.0069$. We cannot legitimately expect the leading order difference equation to be more than qualitatively accurate.

A more positive aspect is obtained by dividing the diff :rence between computed and predicted cusp locations by $\delta \ln (1 / \delta)$ : we should find that these ratios tend (for constant $\rho$ ) to an $O(1)$ constant as $\sigma \rightarrow \infty$. The results of this are given in table II, and are e'sen better t'ian one might hope for, thus reinforcing our opinion that the present analysis captures the essential dynamics. 
Table II

Values of $R_{s}$ are the ratio of the difference between computed and predicted values of $C_{s}$ in table $I$, and $\delta \ln (1 / \delta)$, where $\delta=b / \sigma$, $b=1$, for the sth cusp.

\begin{tabular}{lrrrrr}
\hline$\rho$ & $\boldsymbol{r}$ & $\sigma$ & $\boldsymbol{R}_{1}$ & $\boldsymbol{R}_{2}$ & $\boldsymbol{R}_{\mathbf{3}}$ \\
\hline 1.1 & 110 & 100 & 1.396 & & \\
1.1 & 330 & 300 & 1.404 & & \\
1.1 & 550 & 500 & 1.416 & 1.27 & 1.10 \\
1.2 & 60 & 50 & 1.355 & & \\
1.2 & 360 & 300 & 1.357 & 1.18 & 0.98 \\
1.2 & 600 & 500 & 1.376 & & \\
\hline
\end{tabular}

\subsection{Height and width of cusps}

We see from (6.4) that the height of each cusp should be $M_{n+1}=2(Z=2 r$, or inore accurately $2(r-1))$, as shown in fig. 7. None of the plots in fig. 8 show this feature, despite taking a variety of different initial values for the variables. To see why this should be so, consider the approximate form of each cusp, (5.18):

$M_{n+1} \approx 2-k\left|\zeta-\zeta_{s}\right|^{\beta}, \quad \zeta_{s}=\left|a_{s}\right|$

from (5.16) $\beta \sim O(\delta)$, whereas from (5.17) $k \sim$ $O(1)$ (in fact $k \approx 0.5$ by inspection). Thus since $\beta \ll 1$ for $\delta \rightarrow 0,\left|\zeta-\zeta_{s}\right|^{\beta} \sim 1$ except when $\left|\zeta-\zeta_{s}\right|$ is extreinely small, i.e. $\left|\zeta-\zeta_{s}\right| \sim \exp [-O(1 / \beta]$. Let us define the width $\Delta_{f}$ of a cusp in the following way. From (6.9), the value of $M_{n+1}$ outside cusps is about $2-k$. We define $\Delta_{f}$ for $0<f<1$ by supposing that a fraction " $(1-f)$ " of the cusp lies inside the interval $\left(\zeta_{s}-\Delta_{f} / 2\right.$, $\left.\zeta_{s}+\Delta_{f} / 2\right) ;$ precisely, when $\zeta=\zeta_{s} \pm \Delta_{f} / 2$, (2$\left.M_{n+1}\right) / k=(1-f)$, from which it follows if $f$ is reasonably small, that we can define

$\Delta_{f}=2 \exp \left[-\frac{f}{\beta}\right]$

Roughly, this means that the inverval of $M_{-n}$ values over which $M_{n+1}$ gets more than a fraction $f$ of the distance up the cusp (whical is $2-k$ ) is $\Delta_{f}$. See fig. 9. For example, the three

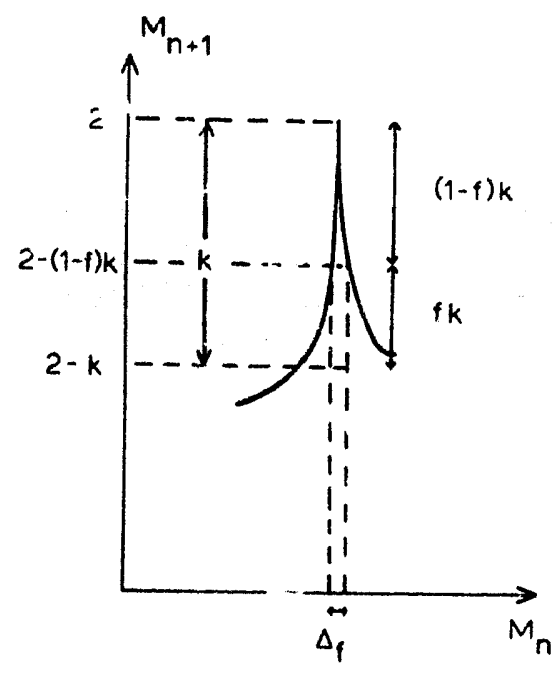

Fig. 9. Definition of the cusp width.

cusps in fig. 8c display points which are up to $\approx 10$ units above the ambient value $\approx 120$, with a theoretical maximum at the cusp tip of $2(r-$ $1) \approx 180$; thus $k \approx 120 / 180, M_{n+1}$ attains $\approx 130 / 180$, so $1-f \approx 50 / 60 \rightarrow f \approx 0.2$. This means that the interval of $M_{n}$ values for which we could expect maxima greater than those on the plot is $\Delta_{f} \approx 2 \exp [-0.2 / \beta]$. Here $\beta \approx 0.02$, and $s(1$ $\Delta_{f} \approx 10^{-4}$, which corresponds to a width $Z \approx 10^{-2}$ in the original units. The interval of values of $M_{n}$ for which $M_{n+}$, is further up the cusp decreases exponentially with $M_{n+1}$. For values of $M_{n+1}$ for which $\left(2-M_{n+1}\right) /(2-k)$ is not close to 1 we have $\left|\zeta-\zeta_{s}\right| \sim \exp (-O(1) / \beta)$, so that (6.10) extends up to $f \sim O(1)$. Thus the top half of the cusp may lie in an interval of $M_{n}$ values of thickness $\Delta_{1 / 2} \approx \exp [-1 / 2 \beta]$, which for fig. 8 $(\beta \approx 0.02)$ is about $\approx 10^{\text {i0 }}, \cdots:=10^{-8}$ in the original units. It is not surpriag that the tops of these cusps are invisible with a numerical experiment such as that reported here. Nevertheless we claim they are there.

It is possible to improve the observed cusp heights by an interval bisection procedure designed to score a direct hit on the cusp. The thinness of the cusps becomes apparent as the top sections are vertical straight lines within graphical accuracy. The height numerically attainable in the cusps is directly limited by the 
precision used. (Fig. 8d features a cusp sharpened in this way.)

\subsection{Prediction of the possible bifurcation behavior}

Prescription of a difference equation for $M_{n}$ "solves" the original problem in the sense that this equation may be used to predict successive values of $M_{n}$, and thus to establish whether the orbit ultimately approtiches a limit cycle or aperiodic behaviour (see, for example, May [11]). Particularly, fixed point: of the difference equation ( $/$ ntersections with $M_{n+1}^{i}=M_{n}$ ) correspond to limit cycles of the orbit: these are stable if $\left|d M_{n+1} / d M_{n}\right|<1$, but become unstable as $\left|d M_{n+1} / d M_{n}\right|$ increases through one, bifurcating successively to doubly, quadruply and higher order periodic solutions before becoming chaotic. This sequence of transitions is shown in fig. 10, and occurs for a mapping such as in fig. 7 when the cusps move to the right, or alternatively the troughs between cusps deepen.

Another kind of transition is shown in fig. 11 . Here the cusps move to the left (or the troughs become shallower) and a stabie liruit cycle and ai! unstable limit cycle in one trough coalesce and disappear. the motion being replaced by an intermittent chacs (Manneville and Pomeau [4]) or by a hard transition to chaotic or periodic behaviour, perhaps localised in another trough.
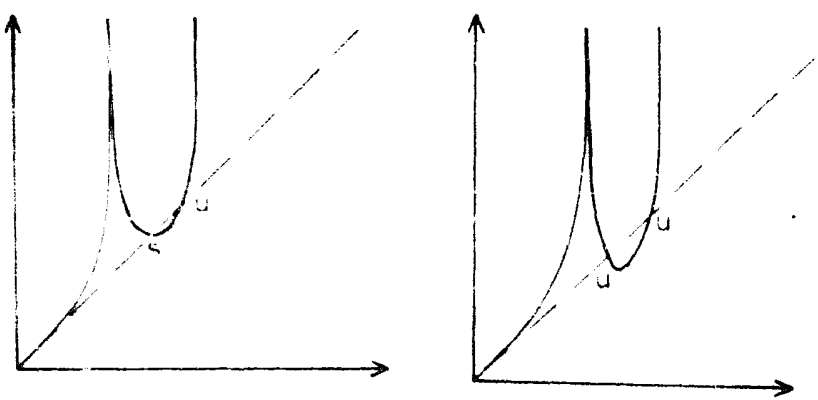

Fig. 10. Schematic bifurcation as the cusps move to the righe (or deepen). A stable (s) periodic solution of (3.1) (fixed point of the mapping) loses stability (u) by period doubling.

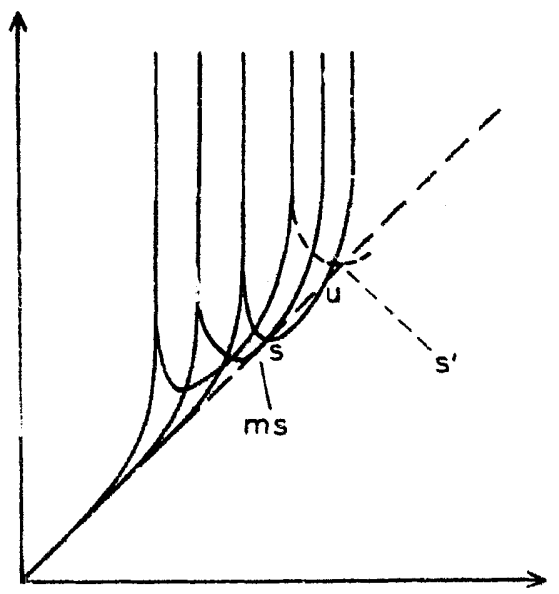

Fig. 11. Schematic bifurcation as the cusps move to the left. A stable (s) and unstable (u) periodic solution coalesce to a marginally stable (ms) periodic solution which then vanishes, and a hard bifurcation occurs; in the case shown this is to a stable $\left(\mathrm{s}^{\prime}\right)$ peri, dic solution in a neighboring trough.

At nigis $\sigma$, the latter appears more likely, as seen in he figure.

\subsection{Effect of $r$ and $\sigma$ on difference equation}

First consider the effect of increasing $r$, and thus $\rho$ at ? fixed high value of $\sigma$. The relevant formulae a:e those for the position of the cusps,

$M_{n}=C_{s}=1+\frac{1}{4 \rho}+\frac{\delta^{2 / 3}(1+4 \rho)^{2 / 3}}{2^{1 /} \rho}\left|a_{s}\right|$,

and the envelope equation determined by (5.7). In terms of $\theta$ given by (5.6), the $s$ th cusp is at

$\theta=\frac{1}{4 \rho}+\frac{\delta^{2 / 3}(1+4 \rho)^{2 / 3}}{2^{2 / 3} \rho}\left|a_{s}\right|$

As $\rho$ increases through 1 , the fixed point $\phi=0$ (corresponding to $Z=r-1$ ) becomes unstable, and may bifurcate either to aperiodic or periodic motion. At high $\sigma$, it usually moves to a limit cycle corresponding to a fixed point. of $\phi$ in the first trough (as in fig. 8). As $\rho$ increases, two things happen. From (6.11), the cusps all move 
to the left; also, (5.7) suggests (since apparently $\phi>\theta$ for $\theta<1 / 4 \rho$ ) that the envelope equation is slightly flattened out as $\rho$ increases, so that the depih of the troughs will be slightly increased. These are opposing effects, so it is not transparent what will occur, and this may we!l depend on the precise value of $\sigma$. (6.5) suggests that at higher values of $\delta$, the decay of the envelope curve for $\theta>1 / 4 p$ will be more rapid, so that the troughs will be shallower, and th: higher order cusps will be less relevant to ti.e dynamics. Also, in this case the shift of the cusps with $\rho$ is more pronounced, so we might expect the shift to be the dominant effect, in which case we might expect intermittent transition between periodic solutions in one trough to periodic or aperiodic solutions in an adjoining trough. This $\mathrm{f}(\mathrm{r}$ example occurs in fig. 12, where plots for $\sigma=100$ at various values of $r$ are given. At $r=110$, a stable limit cycle $(Z \approx 147)$ exists in the first trough, whereas at higher values, this bifurcates to a stable limit cycle in the second trough (e.g., $Z \approx 179$ when $r=130$ ). This set of results is commented on further in 6.8 below.
As we increase $\sigma$ at fixed $\rho$ (not $r$ ), the only sensible difference is in the spacing and the width of the cusps. The spacing is $O\left(\delta^{2 / 3}\right)$, whereas the width is $\Delta_{f} \approx \exp [-f / \delta]$; thus at $\sigma=10^{3}$, the spacing is $\approx 10^{-2}(\approx 0(10)$ in original units) whereas $\Delta_{0.01}=10^{-4}\left(0\left(10^{-1}\right)\right.$ in original units). Therefore as $\delta \rightarrow 0$, the cusp spacing decreases algebraicaliy, whereas the cusp wiath decreases exponentially, and the form of the difference equation will be as in fig. 13a, where a multitude of spikes are superimposed on the underlying envelope equation (5.7). If $\phi^{\prime \prime}>0$ for $\theta<1 / 4 \rho$ (as is indicated by the numerical results), then $\phi$ is convex $f \wedge r \phi<1 / 4 \rho$, concave for $\phi>1 / 4 p$, and the envelope equation has a unique stable fixed point. Therefore, as $\sigma \rightarrow \infty$, the motion will become periodic, except for particular values of $\rho$ for which this fixed point "coincides" with (is within $\exp [-\sigma(1) / \delta]$ of) one of the cusps. The solutions would then be chaotic, being cumposed of "slow" sequence of maxima of $\mathrm{Z}$ which approach the fixed points of the envelope equation, followed by a rapid ejection as the sequence encounters the cusps. This is visualised in fig. 13b.

The sequence of transitions as $\rho$ increases at

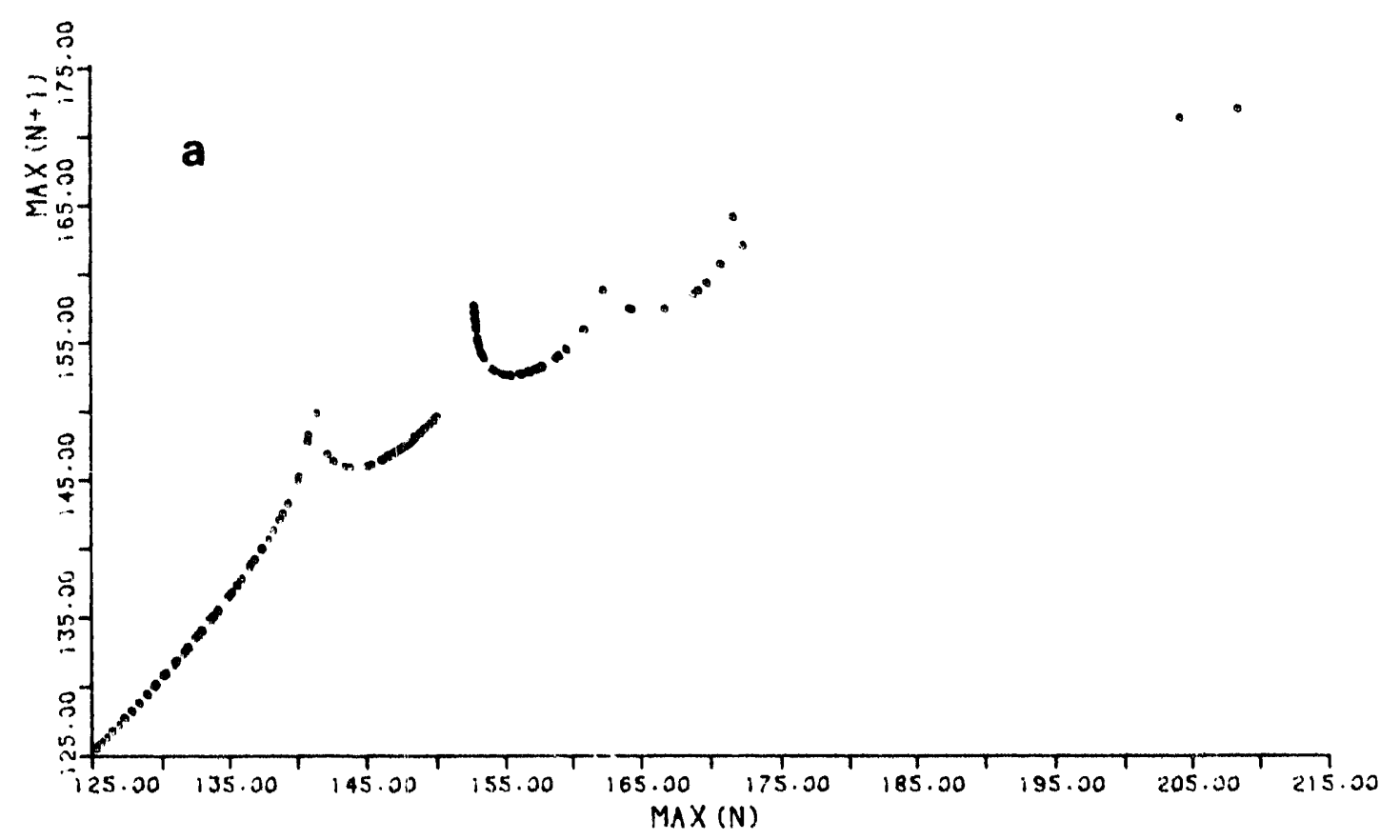



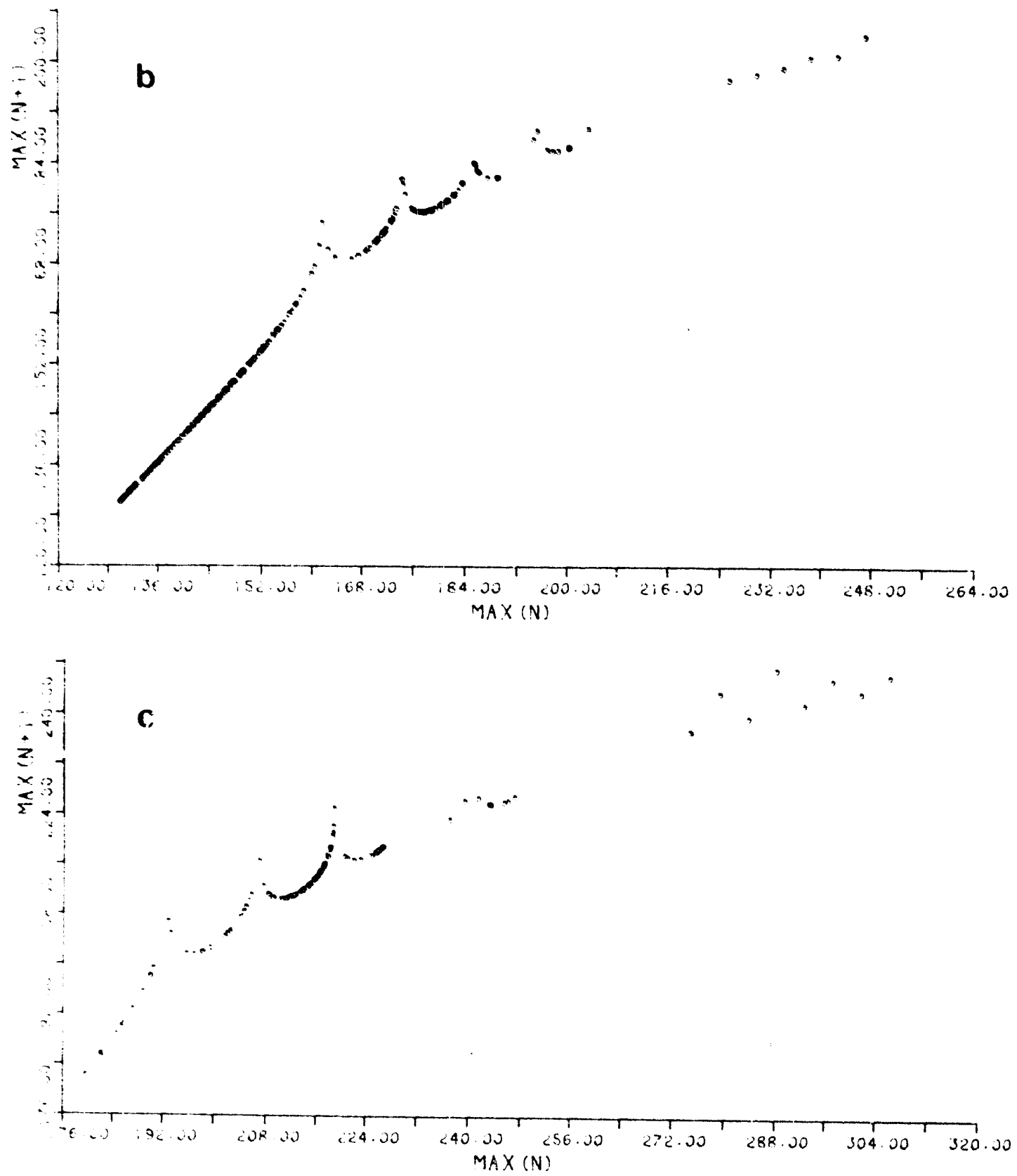

Fig. 12. Numerical plots of the difference equation at $b=1, \sigma=100$, and (a) $r=110 ;$ (b) $r=130 ;$ (c) $r=160$.

such high values of $\sigma$ would be between limit cycles $L_{s}$ corresponding to fixed points of $\phi(\theta)$ in the $s$ th trough, where $L_{s}$ occurs for $\rho_{s}<\rho<$ $\rho_{1}, 1$, and $\rho_{s}, s \geqslant 1$, is the value of $\rho$ for which the fixed point of $\phi$ coincides with the $s$ th cusp. These transitions would be "fuzzy", in the sense that the period doubling path to chaos would occur in an interval $\left|\rho-\rho_{s}\right|=o(1)$ (actually $C(\delta))$, whereas $\left|\rho_{s}-\rho_{s-1}\right|=\mathcal{O}\left(\delta^{2 / 3}\right)$.
The period of such limit cycles is apf oximately given by $r^{*}$, and thus, from (6.1) and (6.3),

$$
\tau^{*}=\frac{(1+4 \rho)^{1 / 2}}{\left[(1+4 \rho)^{1 / 2}-1\right]}\left[2(1-\ln 2)+\ln \left(\frac{M_{n}}{+1 / 4 \rho}\right)\right] .
$$

As $\rho$ increases through $\rho_{s}$, the period and am- 


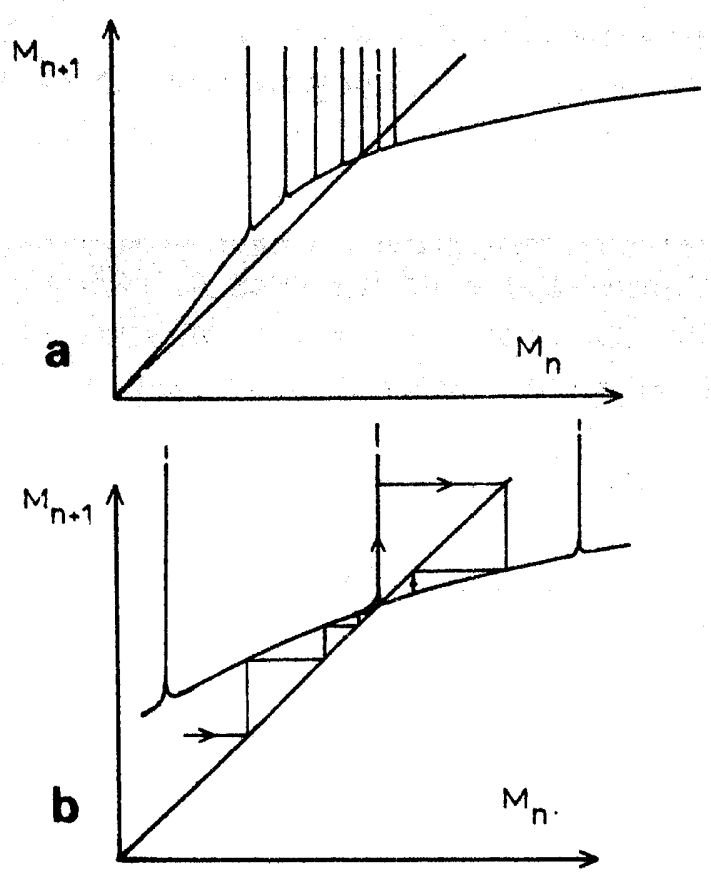

Fig. 13. (a) Schematic form of difference equation at very high $\sigma:$ the envelope equation is overlain by a sequence of spikes. (b) Illustration of chaotic motion when a spike overlies the fixed point of the envelope difference equation.

plitude of the oscillation is continuous, and transition is only grossly apparent in the different number of zeros of $x$ and $y$ between maxima of $z$ (see also (6.8)), which increases by one at each transition.

The results at lower $\sigma$ are typified by the results of Lorenz [3]. In his paper, Lorenz had $b=$ $8 / 3, r=28$ and $\sigma=10$, so $\delta \approx 1 / 4$ and $\rho \approx 2.8$. He found one cusp at $Z \approx 38.5$ with a height of 48.3 . In our notation, the cusp was at $M_{n}=38.5 / 28 \approx$ 1.38 , with a maximum of $M_{n+1}=48.3 / 28 \approx 1.73$. The maximum is $O(\delta)$ below 2 , as might be expected from section $4 .(6.11)$ predicts that the first cusp $C_{1}$ should be at

$$
C_{1}=1+\frac{1}{4 \rho}+\frac{\delta^{2 / 3}(1-4 \rho)^{2 / 3}}{2^{4 / 3} \rho} \cdot\left|a_{1}\right| .
$$

With $\rho=2.8, \delta=4 / 15,\left|a_{1}\right|=2.338 \ldots$, we ind that $C_{1} \approx 1.82$, which is an over-estimate by about $O(\delta)$ (in fact by $1.24 \delta \ln (1 / \delta)$ ). More im- portantly, we note that a second cusp is not apparent, and the first trough does not "turn around". To understand this, consider again the difference equation (5.9). We find a differential equation for $M_{n+1}$, as in (5.7); the result is

$$
\begin{aligned}
& \frac{d M_{n+1}}{d M_{n}}=\frac{\left(2-M_{n+1}\right)\left[1+\frac{2^{7 / 3} p \delta^{1 / 3} \mathrm{Ai}^{\prime}(-\zeta)}{(1+4 \rho)^{2 / 3} \mathrm{Ai}(-\zeta)}\right]}{M_{n}\left[(1+4 \rho)^{1 / 2}\left\{1-\frac{2-M_{n+1}}{(1+1 / 4 \rho)}\right\}^{1 / 2}-1\right]} \\
& M_{n}=1+\frac{1}{4 p}+\frac{(1+4 \rho)^{2 / 3} \delta^{2 / 3}}{2^{4 / 3} \rho} \zeta .
\end{aligned}
$$

Although formally, minima $c: M_{n+1}$ occur close to the precading cusp (when $\mid \mathrm{Ai}\left(-\zeta_{i}^{i} \sim \delta^{1 / 3}\right.$ ), it is apparent that this is hardly accurate for Lorenz' parameters, where for example $2^{-1 / 3} \rho \delta^{1 / 3} /(1+$ $4 p)^{2 / 3} \approx 1.6$ then the minimum in the first trough is a distance $\zeta \sim 1$ from the first cusp, and hence a distance $M_{n} \sim(1+4 \rho)^{2 / 3} \delta^{2 / 3} / 2^{4 / 3} \rho$, numerically of $O(1)$. At slightly larger values of $\sigma$, say $\delta \approx$ $1 / 10$ to $1 / 20$, we expect the same to be qualitatively true: that is, the increased spacing of the cusps causes the troughs to be deeper than their counterparts at very high $\sigma$. Thus, reducing $\sigma$ moves the cusps to the right, and causes the troughs to deepen. This induces the kind of transition to shaos through period doubling envisaged in fig. 10 .

\subsection{The limit $r \rightarrow \infty$}

We discuss this here because of the other analytic work in the case $r \rightarrow \infty, \sigma \sim 1$ (Robbins [18], Shimizu [19]). At fixed $\sigma$, there is a stable limit cycle as $r \rightarrow \infty$. Our analysis is only valid for $\rho \sim 1$; if we consider larger and larger $\rho$ (with $\sigma \gg 1$ ), we still obtain the spiky dirference map aiscussed in section 6 , so although this gives a limit cycle for "most" $\rho$, it is rot equivalent to the limit cycle for $r \rightarrow \infty, \sigma \sim 1$, which is not a relaxation type oscillation, as those discussed here are. Further, numerical results (Morioka and Shimizu [6]) suggest that the cusp features of the difference map dis- 
appear at higher $r$ (between 200 and 300 for $a=10$. $b=4$ ). Therefore there is a question as to how the two parameter regimes $r \sim \sigma \gg 1$ and $r \gg 1, \sigma \sim 1$ join together, and whether there might be some distinguished intermediate limit which is necessary to join them. In fact, recent work of Sparrow [28] indicates that these two different sclution types may simply be different aspects of the system behaviour. When $r \gg 1$ $(\sigma-1)$, anomalous (but unstable) orbits can exist in addition to the stable periodic orbit provided $b$ (and hence $\delta$ ) is small enough. These anomalous orbits are the analogues of the relaxational orbits considered here: as $\sigma$ increases. the stable periodic orbit becomes a constituent of some of these anomalous orbits. and consequently they attain stability. Conversely, as $\rho$ increases in (4.2), then the solution of (4.8) alters in form: $y$ becumes large, and the fast solution oscillates on a time scale $t \approx 1 / \sqrt{\rho}$ : because of this, the damping term in (4.8) is relatively small, and becomes comparable to the others neglected in deriving (4.8); thus the scaling of the present paper becomes irrelevant, and the disappearance of the cusp at high $r$ is a manifestation of this (the anomalous orbits exist, but are no longer stable at high $\rho$ ).

\subsection{Nature of the solutions}

Lastly, we consider the nature of the solutions, and the precise nature of the cusps. In fig. 14, we show the solutions versus time at $r=$ $110, \sigma=100$ (the corresponding differe nce map is shown in fig. 12) with initial values $X=Y=$ $12, Z=125$. The solution grows until it appears to settle into a periodic oscillation (this is not quite evident in fig. 14) with successive maxima
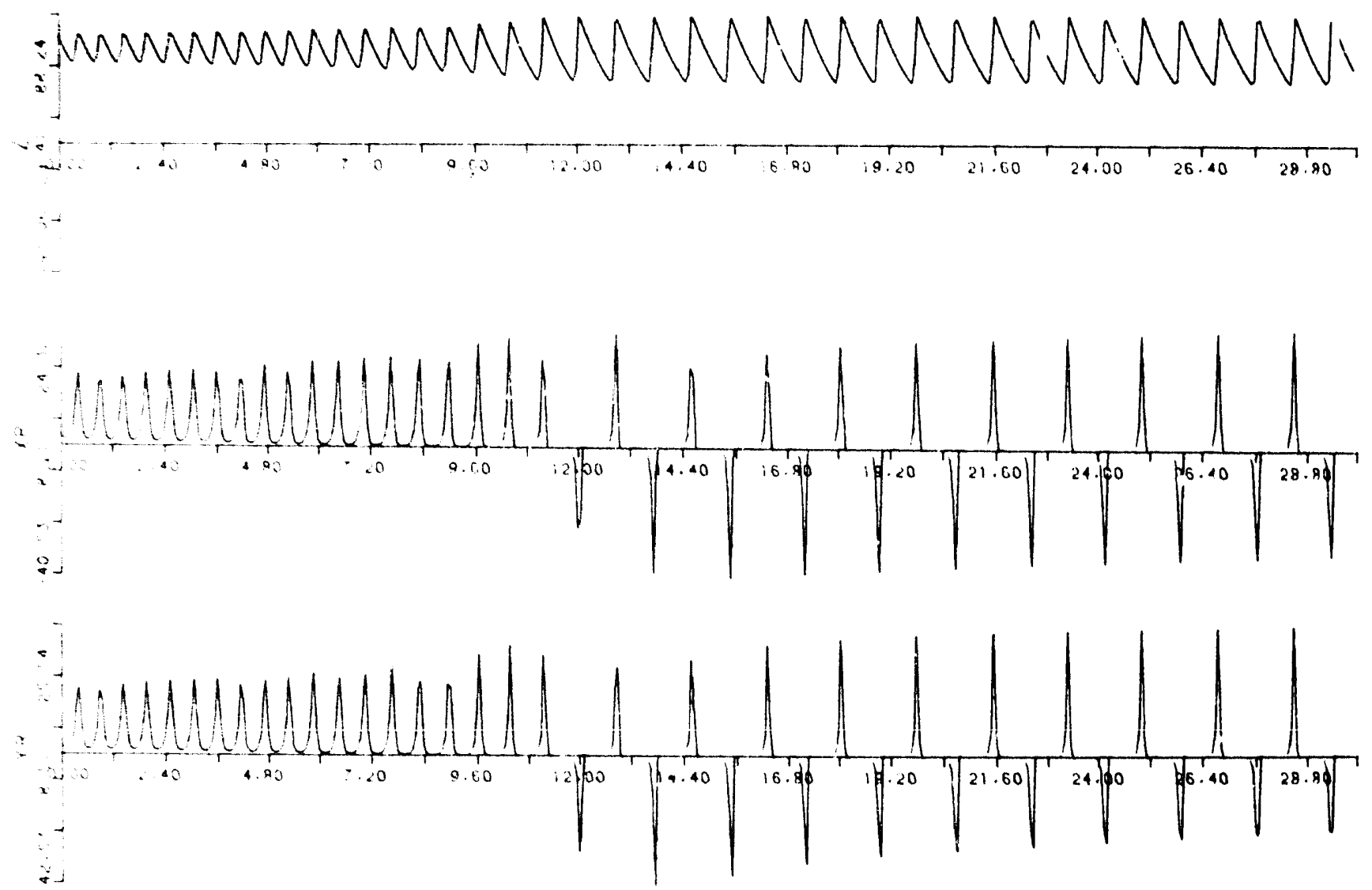

Fig. 14. Numerical solution of the Lorenz equations; $r=110, \sigma=100, b=1$, initi 1 values $X=Y=12, Z=125$. 
of $Z \approx 147$. In the initial phase, $0 \leqslant T \leqslant 11.0, X$ and $Y$ are one-signed and maxima of $Z$ lie on the left of the first cusp; for $T \geqslant 11.0$, the maxima lie in the first trough, and $X$ and $Y$ have exactly one zero between maxima.

We contrast $t$ is behaviour with that shown in fig. 15. These are time plots of $X, Y$ and $Z$ at the same parameter values, but with initial conditions $X=1, Y=Z=0$. Such a condition, very near the origin, gives a very high first maximum, and is thus able to direct the solution off the rising branch of the first cusp. In fact we see (particularly from the $X$ and $Y$ plots) that the solution behaves erratically; closer examination of the numerical solutions shows that $X$ and $Y$ have exactly two zeros between maxima, and that these maxima lie on the second trough of fig. 12a. Actually, the last two groups of eight pulses in fig. 15 are similar; this may represent an approach to an eight-cycle of the difference map, or it may be transitory behaviour.

This shows that multiple dynamic states are realisable at one set of parameter values (note that exactly the same phenomenon occurs in fig. 8d; we also see that motions in each trough are characterised by different numbers of zeros of $x$ and $y$ between maxima of $z$ : in the first trough there is one zerc in the second there are two, and so on. The reason for this lies in the matching procedure between the fast and slow phases. In fact, consideration of the slow solution (4.56), with $B$. given by (4.46) and (4.48), shows that for $\zeta<\left|a_{1}\right|$, the first zero of $\mathrm{Ai}(-\zeta), x$ (and so also $y$ ) docs not cross the axis between maxima of $z$ : this corresponds to $T \leqslant 11$ in fig. 14. For $\left|a_{1}\right|<\zeta<\left|a_{2}\right|$ (the first trough), there is exactly one zero of $x$ and $y(T \geqslant 11)$; in the second trough, $\left|a_{2}\right|<\zeta<\left|a_{3}\right|$, there are exactly two

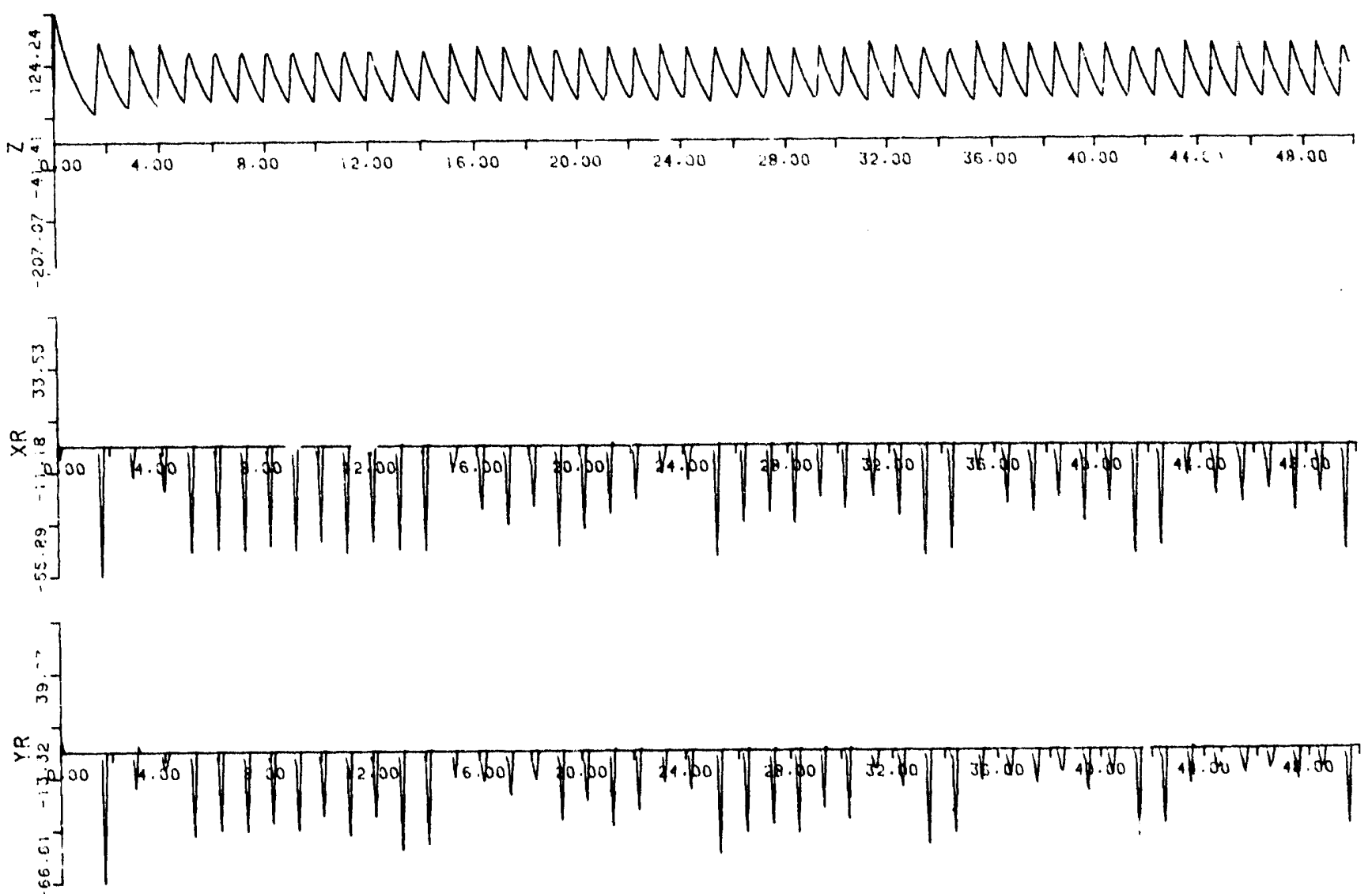

Fig. 15. Numerical solution of the Lorenz equations; $r=110, \sigma=100, b=1$, initial values $X=1, Y=Z=0$. 
zeros between pulses, so the successive peaks of $x$ and $y$ are of the same sign. For solutions in higher troughs (which may be obtained at higher values of $\rho$ and $\sigma$ ), the number of zeros increases accordingly.

We note that this behaviour requires that we do not take a variable mesh in the numerical integration. even in the slow phase. since otherwise we might lose the delicate oscillatory nature of $x$ and $y$ in these phases. If $h$ is the time step in $T$ used, then sufficient accuracy is probably obtained. from (3.5), provided

$h \leqslant \frac{0.1}{a}$.

with a suitable difference approximation. An upper limit for accuracy purposes may be $\approx 0.3 / \sigma$. The step size used in computing fig. 15 $(\sigma=100)$ was $h=0.1 / \sigma=10^{3}$.

\section{Discussion}

We have analysed the behaviour of the Lorenz equations in the limit in which the Prandtl number. $\sigma$, is large, and in which the Rayleigh number $r=C(\sigma)$. In this situation, th: colutions behave in a relaxational manner. and an approximate description of both fast and slow phases is possible. We obtain an explicit description of the difference equation relating successive maxima of $z$, and find that as $\sigma \rightarrow \infty$, this curve will have a multitude of cusps, spaced at intervals $\approx \delta^{2 / 3}(\delta=b / \sigma)$ and of "width" expl- $C(1) / \delta]$. The curve thus essentially consists of a smooth (monotone increasing) envelope, with superimposed narrow bands, in which the cusps are situated (see fig. 7). As $r$ increases at a fixed value of $\delta \ll 1$, the bifurcation sequence is most likely to consist of successive "hard", possibly "intermittent" bifurcation of stable sei iodic limit cycles (in one trough) to motions in neighboring troughs periodic or chaoitic solutione. As $r$ increases further, chaotic (or long period) solutions may simplify by decreasing period (period doubling as $r$ decreases), until eventually the "fundamental" periodic solution in the trough becomes stable, and further intermittent or hard bifurcation may occur. This agrees with the results of Manneville and Pomeau [4] and Morioka and Shimizu [6]), and shows how F rriod doubling and intermittency (soft and hard bifurcation) may be understood in terms of an appropriate pictorial diagram.

Although we can associate much oi ine rich behaviour of the Lorenz equation with a single difference equa.ion, it is not so easy to classify a!l the tyres of behaviour, $o$ possible regimes which may occur. One view of the behaviour is that for any particular set of parameters, there are many "fundamental" periodic solutions (by which we mean intersections of the difference equation with the line $M_{n+1}=M_{n}$, i.e., fixed points of the map $\left.M_{n} \rightarrow M_{n+1}\right)$; as $r$ ircreases the stability of these changes, and consequently the orbits flit from one periodic orbit to the next.

The nature of the approximate analysis is the following; the slow phase is (in principle) acurate to $\exp [-O(1) / \delta]$ but the fast phase is accurate only to $O\left(\delta^{2} \ln ^{2}(1 / \delta)\right)$, consequently this is the error in determining tise difference equation. For mc st purposes, we have considered only the leading; order approximation, valid to terms of $O(\delta \ln (1 / \delta))$ : the higher approximation may be more trouble than it is worth.

Multiple cusp behaviour has also been observed by Lorenz [26] at lower values of $b$. down to $b=1 / 8$. His choice of parameters may be written, for small $b$, as

$$
\begin{aligned}
& r=r_{\mathrm{cm}}=9\left[1+\frac{2}{3} b-\frac{b^{2}}{72} \cdots\right], \\
& \sigma=\sigma_{\mathrm{m}}=3\left[1+\frac{5 b}{6}-\frac{b^{2}}{48} \cdots\right],
\end{aligned}
$$

and thus

$$
\begin{aligned}
& \rho=3\left[1-\frac{b}{6}+\frac{7}{48} b^{2} \cdots\right], \\
& \delta=\frac{1}{3} b\left[1-\frac{5}{6} b+\frac{103}{144} b^{2} \ldots\right] .
\end{aligned}
$$


At $b=1 / 8$, for mxample, $\delta \approx 0.04$, and the previous ideas shurld be valid. However, in this case, $\gamma$ in (3.7) is given by $\gamma=1 / \sigma \approx$ $\frac{1}{3}[1-5 b / 6 \cdots] \sim O(1)$, and no simple first integra! of (3.7) such as (4.6) exists; nevertheless the ideas of the analysis should go through; the study of the slow phase is similar, and thus the oniy obstacle to an explicit formula in this case is the attuinment of a formula relating a minimum of $z(\dot{z} \rightarrow 0$ as $t \rightarrow-\infty)$ to the subsequent maximum $(z \rightarrow 0$ as $t \rightarrow+\infty)$ through solution of the fast phase equations

$$
\begin{aligned}
& \dot{x}=\wedge+y, \\
& \dot{y}=\rho x(1-z)-\gamma y, \\
& \dot{z}=x y .
\end{aligned}
$$

It has now become established (Robbins [29], Kaplan and Yorke [30]) that the onset of observable chaotic behaviour takes place away from the sub-critical Hopf bifurcation of the .cn-trivial steady states, and is due to the establishment of a strange invariant set of periodic and aperiodic orbits at a parameter value at which there exists a homoclinic orbit. In terms of the present context, it is clear that a homoclinic orbit is the limit of a slow pulse-fast pulse-slow pulse solution in which $m_{n} \rightarrow 0$; on the difference map this corresponds to the existence of a cusp, and we can thus identify with each cusp a homoclinic orbit: in fact, we find that there can be a large number of such homoclinic orbits, each having a different 'crossing number' (number of zeros of $x$ between maxima of $z$ ), which may also be thought of as the number of twists round the $z$-axis: each new homoclinic orbit adds in another twist.

From our analysis, we can predict that the Hopf bifurcation occurs for $r \sim \sigma$ as $\sigma \rightarrow \infty$ (this is obvious); moreover, the first homoclinic 'explosion' (to use Sparrow's [28] terminology) takes place when the first cusp first intersects the line $M_{n+1}=M_{n}$. At leading order, this is when $\rho=\frac{1}{4}$, i.e. $r \sim 0.25 \sigma$ as $\sigma \rightarrow \infty$ : this is roughly in line with Robbirs' [29] result. Since the homoclinic bifurcation takes place for $r$ less than the Hopf bifurcation, one could in principle have a situation where chaotic behaviour is observed, but no Hopf bifurcation at all takes place as the bifurcation parameter is varied. The point is, that the road to turbulence (at least in the Lorenz system) occurs via a homoclinic bifurcation, and the rolle of the Hopf bifurcation is a secondary one of merely soaking up two of the orbits produced by the explosion. In view of the current vogue for thinking of turbulence (particularly, for example, Taylor vortex flow) in terms of 'Hopf-like' bifurvation to higher dimensional tori, it is worth bearing the above points in mind. Particularly, we may recall our introductory remarks on turbulent plane Poisi uille flow, where transition is observed at $\operatorname{Re}($ Reyno:ds number) $\approx 1000$, but the steady state undergoes sub-critical Hopf bifurcation at $\mathrm{Re} \approx 5772$. It may not in principle be worthwhile trying to 'join' the two phenomena together.

\section{References}

[1] $O$. Reynolds, On the experimental investigation of the circumstances which determine winether the motion of water shall be direct or sinuou; and the law of resistance in parallel channels. Phil. Trans. Roy. Soc. 174 (1883) 935-982.

[2] S.A. Orszag and A.T. Patera, Subcritical transition to turbulence in plane channel trows. Phys. Rev. Lett. 45 (1980) 989-993.

[3] E.N. Lorenz, Deterministic non-periodic flow. J. Atmos. Sci. 20 (1963) 130-141.

[4] P. Manneville and Y. Pomeau, Different ways to turbulence in dissipative dynamical systems. Physica ID (1980) 219-226.

[5] I.J. Wygnanski and F.H. Champagne. On transition in a pipe. Part I. The origin of puffs and slugs and the flow in a turbulent slug. J. Fluid Mech. 59 (1973) 281-335.

[6] N. Morioka and T. Shimizu, Transition between periodic and turbulent states in the Lorenz model. Phys. Lett. 66A (1978) 447-449.

[7] J.B. McLaughlin and P.C. Martin, Transition to turFulence in a statically stressed fludl system. Phys. Rev. A12 (1975) 186-203.

[8] J.T. Stuart, On the non-linear mechanics of wave disturbances in stable and unstable parallel flows. J. Fluid Miech. 9 (1960) 353-370. 
[0] K. Stewartson and J.T. Stuart. A nonlinear instability theory for a wave system in plane Poiseuille flow. $J$. Fluid Mech. 48 (1971) $529-545$.

[10] TV. Li and J.A. Yorke. Period three implies chaos. Amer. Math. Monthly 82 (1975) 985-992.

(III) R.M. May. Simple mathematical models with very complicated dynamiss. Nature 261 (1976) 459-467.

[12] M. Henon and $Y$. Pomeau. Two strange attractors with a sirmple structure. In: Turbulence and Navier Stokes equations. A. Dold and B. Eckmann. eds. (Springer. Berlin. 1976) pp. 29-68.

[13] S. Rosenblat and S.H. Davis. Bifurcation from infinity. SIAM J. Appl. Math. 37 (1979) 1-19.

(14) H. Schlichting. Boundary layer tneory, (McGraw-Hill, New York 1979).

[15] T. Shimizu and N. Morioka. Chaos and limit cycles in the Lorenz model. Phys. Lett. o6A (1978) 182-184.

[16] N.V. Roshchin. Unsafe stability houndaries of the l.orentz (sic) model. PMM 42 (1978) 950-952. In translation. pp. 1038-1(1)41.

(1) P. Manneville and Y. Pomeau. Intermittency and the Lorenz model. Phys. Lett. 75A (1979) 1-2.

[18] K.A. Robbins. Periodic solutions and bifurcation structure at high $R$ in the Lorenz model. SIAM J. Appl. Math. 36 (1979) 457-42.

[19] T. Shimizu. Analytic form of the simplest limit cycle in the Lorenz model. Physica 97A (1979) 383-398.

[20] J.A. Yorke and F.D. Yorke. Metastable chaus: the transition to sustained chaotic behaviour in the Lorenz model. J. Stat. Phys. 21 (1979) 263-277.

[21] J.D. Cole, Perturbation methods in ann: mathematics (Waltham, Blaisdell. Ma, 1968).

[22] A.C. Fowler. An asymptotic analysis of the logistic delay equation IMA J. Appl. Math. 28 (1982) 41-49.

[23] H. Cheng and T.T. Wu, An aging spring, Stud. Appl. Math. 49 (1971) 183-185.

[24] M. Abramowitz and A. Stegun, Handbook of mathematical functions (Dover, New York, 1968).

[25] C.J. Marzec and E.A. Spiegel, Ordinary differential equations with strange attractors. SIAM :. Appl. Math. 38 (1980) 403-421.

[26] E.N. Lorenz, On the prevalence of aperiodicity in simple systems. In: Global Analysis, M. Grrela and J.E. Marsden, eds. (Springer, Berlin, 1979) pp. 53-75.

[27; j. Kevorkian and J.D. Cole, Perturbation methods in applied mathematics (Springer, New York, 1981).

[28: C.T. Sparrow. Bifurcations in the Lorenz equations. Springer Lecture Notes in Applied Mathematics, to appear, 1982.

[29] K.A. Robbins, A new approach to subcritical instability and turbulent transitions in a simple dynamo. Mr h. Pruc. Camb. Phil. Soc. 82 (1977) 309-325.

[30] 8.L. Kaplan and J.A. Yorke, Preturbulerice: a régime observed in a fluid flow model of Lorenz. Comm. Math. Phys. 67 (1979) 93-108. 